Analytic approach to nonlinear

hydrodynamic instabilities driven by time-dependent accelerations

K. O. Mikaelian

October 6, 2009

Physical Review E 
This document was prepared as an account of work sponsored by an agency of the United States government. Neither the United States government nor Lawrence Livermore National Security, LLC, nor any of their employees makes any warranty, expressed or implied, or assumes any legal liability or responsibility for the accuracy, completeness, or usefulness of any information, apparatus, product, or process disclosed, or represents that its use would not infringe privately owned rights. Reference herein to any specific commercial product, process, or service by trade name, trademark, manufacturer, or otherwise does not necessarily constitute or imply its endorsement, recommendation, or favoring by the United States government or Lawrence Livermore National Security, LLC. The views and opinions of authors expressed herein do not necessarily state or reflect those of the United States government or Lawrence Livermore National Security, LLC, and shall not be used for advertising or product endorsement purposes. 


\title{
Analytic approach to nonlinear hydrodynamic instabilities driven by time-dependent accelerations
}

\author{
Karnig O. Mikaelian \\ Lawrence Livermore National Laboratory, Livermore, California 94551
}

We extend our earlier model for Rayleigh-Taylor and RichtmyerMeshkov instabilities to the more general class of hydrodynamic instabilities driven by a time-dependent acceleration $g(t)$. Explicit analytic solutions for linear as well as nonlinear amplitudes are obtained for several $g(t)^{\prime}$ s by solving a Schrödinger-like equation $d^{2} \eta / d t^{2}-g(t) k A \eta=0$ where $A$ is the Atwood number and $k$ is the wavenumber of the perturbation amplitude $\eta(t)$. In our model a simple transformation $k \rightarrow k_{L}$ and $A \rightarrow A_{L}$ connects the linear to the nonlinear amplitudes: $\eta^{\text {nonlinear }}(k, A) \sim\left(1 / k_{L}\right) \ln \eta^{\text {linear }}\left(k_{L}, A_{L}\right)$. The model is found to be in very good agreement with direct numerical simulations. Bubble amplitudes for a variety of accelerations are seen to scale with $S$ defined by $s=\int \sqrt{g(t)} d t$, while spike amplitudes prefer scaling with displacement $\Delta x=\int\left[\int g(t) d t\right] d t$.

PACS numbers 47.20.-k, 52.35.Py 


\section{INTRODUCTION, MOTIVATION, AND NOTATION}

Fluids undergoing accelerations are subject to hydrodynamic instabilities whereby small deviations from a perfect symmetry (such as planarity or sphericity) amplify with time and eventually lead to mixing between the fluids. Best known examples are the Rayleigh-Taylor (RT) [1] and the Richtmyer-Meshkov (RM) [2] instabilities induced by a constant and an impulsive acceleration, respectively. In this paper we study instabilities induced by a time-dependent acceleration $g(t)$.

We are interested primarily in inertial confinement fusion (ICF) capsules where materials and drives are chosen judiciously to minimize hydrodynamic instabilities [3]. Although the implosion proceeds mainly by a series of shocks and constant accelerations, there are periods where the acceleration is not constant. A study of how fluid interfaces evolve during time-varying accelerations may help design more robust capsules.

A second motivation is the following: RT and RM instabilities are idealized special cases of this more general class of $g(t)$ in that they are characterized by a single parameter: The constant $g$ in the case of the RT instability, and the jump velocity $\Delta \mathrm{V}$ in the case of the RM instability. These are, of course, idealizations almost never realized in actual experiments that start with $g=0$ and later reach $g=$ const. Similarly, impulses have a finite width in time before inducing the jump velocity $\Delta \mathrm{v}$. The start-up and pulsewidth issues are ignored in the classical RT and RM results.

A third reason for this study is that time-dependent effects appear even in experiments designed principally for constant accelerations, as in the original rocket-rig experiments on turbulent mixing by RT instabilities [4]. More recently, experiments have 
been performed $[5,6]$ with several deliberately chosen time-dependent $g(t)^{\prime} s$. These experimental profiles for $g(t)$ will form our starting point. Although the experiments had random multimode perturbations and measured turbulent mixing widths, we shall consider single-scale perturbations as in the experiments of Jacobs et al. [7-9]. A firm understanding of the single-scale problem is needed before tackling the much more challenging problem of turbulent mix.

We consider primarily the bubble amplitude, denoted by $\eta(t)$, from its linear regime where $\eta k<<1$ to the highly nonlinear regime $\eta k \geq 1$. Here $k=2 \pi / \lambda$ in plane geometry often called 2D (two dimensional) with $\lambda$ =wavelength of the perturbation, and $k=\beta_{1} / R$ in 3D (three dimensional) or tubular flow with $R=$ radius of the tube and $\beta_{1} \approx 3.832$, the first zero of $J_{1}$, the Bessel function of order one. The initial perturbation has the form $\eta_{0} \cos (k x)$ in $2 \mathrm{D}$ and $\eta_{0} J_{0}\left(\beta_{1} r / R\right)$ in 3D where $\eta_{0} \equiv \eta(t=0)$ is the initial value of the bubble amplitude, taken positive in our convention. Spikes, meaning the penetration of the heavier fluid into the lighter one, will be negative. As before [10] we shall use the parameter $C$ with values $C=2$ for $2 \mathrm{D}$ and $c=1$ for 3D. Like most experiments [4-8] our direct numerical simulations with the hydrocode CALE [11] will be in planar 2D geometry, although we have also performed “3D” axisymmetric tubular flow simulations.

In the linear regime no model is needed because the equation

$$
\ddot{\eta}-g k A \eta=0
$$

describes the evolution of the bubble (and also the spike) for arbitrary $g(t)$. Here $A$ is the Atwood number, $\left(\rho_{B}-\rho_{A}\right) /\left(\rho_{B}+\rho_{A}\right)$, where $\rho_{B}\left(\rho_{A}\right)$ is the density of the 
heavy (light) fluid, and $g$ is directed from fluid A to fluid B. Although Eq. (1) has been applied to the RT and RM cases only, it is valid for any $g(t)$. The limitation to the linear regime, however, severely restricts the use of Eq. (1): $\eta(t)$ rapidly enters the nonlinear regime or, even worse, many experiments start with a weakly nonlinear $\left(\eta_{0} k \sim 1\right)$ amplitude and grow from there, necessitating the use of a nonlinear model.

The solutions to Eq. (1) for the classical RT and RM cases are:

$$
\eta(t)=\eta_{0} \cosh (\gamma t)
$$

and

$$
\eta(t)=\eta_{0}+\dot{\eta}_{0} t=\eta_{0}(1+\Delta \mathrm{vkAt})
$$

respectively [1,2], with $\gamma \equiv \sqrt{g k A}$. For RT we have assumed $\dot{\eta}_{0}=0$ (otherwise a sinh term must be added). For RM $\dot{\eta}_{0}=\eta_{0} \Delta \mathrm{vkA}$, as derived by Richtmyer [2]. Corrections for finite pulse-width are given below. The fact that Eqs. (1)-(3) are independent of $c$ indicates that they apply to both 2D and 3D geometry, with appropriately defined $k$. The nonlinear solutions will be found to depend on $c$. Analytic solutions to Eq. (1) for $g(t)$ other that the above two cases are given in this paper.

In the next section we describe a progression of mathematical approaches needed for the nonlinear evolution of $\eta(t)$. In Sec. III we apply the model to a number of gedanken experiments patterned after the LEM (Linear Electric Motor) experiments of Dimonte and Schneider [5,6], compare model results with CALE simulations, and present predictions for future experiments. In Sec. IV we take up the issue of scaling of bubbles and spikes. Conclusions are given in Sec. V. The Appendix presents mathematical solutions for a number of $g(t)$ 's. 


\section{MODELS AND LEVELS OF ACCURACY}

Computations are used to explain and understand past experiments, as well as predict the outcome of future experiments. We shall present briefly four levels of computational accuracy, starting with the highest level, level 1, which is most accurate but also most complicated, and proceed down to the last level, level 4, which is least accurate but of course very simple.

Level 1. It is generally agreed that the Euler equations expressing conservation of mass, momentum, and energy provide the most accurate description of fluids neglecting the effects of viscosity and dissipation. Momentum conservation, for example, reads

$$
\frac{\partial\left(\rho u_{j}\right)}{\partial t}+\sum_{i=1}^{3} \frac{\partial\left(\rho u_{i} u_{j}\right)}{\partial x_{i}}+\frac{\partial p}{\partial x_{j}}=0
$$

in Cartesian coordinates, $j=1,2,3$ for 3D. Here $\rho$ is the density, $\vec{u}$ the velocity, and $p$

the pressure of the fluid at point $\vec{X}$. The partial differential equations (PDEs) are solved numerically by hydrodynamic codes. We use CALE, an arbitrary Lagrangian Eulerian code [11], to evaluate the various levels discussed below.

Level 2. An assumption, often in the form of a potential, is made to reduce Euler's PDEs to ODEs (ordinary differential equations). We concentrate on Layzer's model [12] because it is widely used, has been successful in the past, and has been generalized by several researchers [13-17]. In its most general form it accounts for time dependent densities, but the two coupled equations (one for the amplitude $\eta(t)$ and the other for the curvature $\eta_{2}(t)$ ) are quite complicated [17]. In this paper we limit ourselves to constant densities for which the equations become 


$$
F_{1} \frac{\ddot{\eta}}{D}+F_{2} \frac{c^{2} k^{2} \dot{\eta}^{2}}{8 D^{2}}+2 g A \eta_{2}=0
$$

and

$$
\eta_{2}(t)=-c k\left\{1+\left[(1+c) \eta_{0} k-1\right] e^{-k(1+c)\left(\eta-\eta_{0}\right)}\right\} / 4(1+c),
$$

with

$$
\begin{aligned}
& F_{1}=2 A \eta_{2}^{2}+c^{2} A k \eta_{2} / 2(1+c)-c^{2} k^{2} / 8(1+c), \\
& F_{2}=2 A \eta_{2}^{2}+(A+c A-2 c-1) k \eta_{2} /(1+c) \\
& +c k^{2}(3 c A / 2+A-c-1) / 4(1+c)^{2},
\end{aligned}
$$

and

$$
D=\eta_{2}-c k / 4(1+c)
$$

As before, $A$ is the Atwood number, $\eta(t)$ is the bubble amplitude, and $\eta_{2}(t)$ is its curvature with initial value $\eta_{2}(0)=-c k^{2} \eta_{0} / 4$. The above equations are derived in the Appendix of Ref. [17].

For a single fluid ( $A=1$ ) Eq. (5) reduces to

$$
\left(2 \eta_{2}+c k / 2\right) \ddot{\eta}+c^{2} k^{2} \dot{\eta}^{2} / 4+2 g \eta_{2}=0
$$

while Eq. (6) remains the same. The $A=1$ results were given in [13] and applied to bubbles only. Zhang proposed using the same $A=1$ equations, with a negative $\eta$, for spikes [14]. The generalization to arbitrary $A$ was achieved by Goncharov [15] and Eq. (5) above reduces to his Eq. (8) for 2D and Eq. (18) for 3D, i.e., for $c=2$ and $c=1$ respectively. 
This model, despite being a relatively high level 2 model, suffers from at least three limitations or failures, reported in [10]. Extending the arbitrary- $A$ model to spikes, as proposed by Goncharov [15], does not work. Even for bubbles, one cannot apply it to just any $g(t)$ - one must maintain the sign (negative) of $\eta_{2}$ [10]. These two failures are related, as changing the sign of $\eta$ and $\eta_{2}$ is equivalent to interchanging bubbles and spikes. A third failure occurs for bubbles with large initial amplitudes $\eta_{0}>\left(\eta_{0}\right)_{\max }$ given by [10]

$$
\left(\eta_{0} k\right)_{\max }=\frac{c}{2(1+c)}\left(1+\sqrt{1+\frac{4(1+c)}{A c^{2}}}\right)
$$

This failure can be remedied in the level 3 model.

Level 3. As noted in Ref. [13], the Layzer model is simplified not for $\eta_{0}=0$, as assumed by Layzer, but by taking $\eta_{0}=\eta^{*}=1 / k(1+c)$. For this value of $\eta_{0}$ one obtains a constant $\eta_{2}$, i.e., $\eta_{2}(t)=\eta_{2}(0)=-c k^{2} \eta_{0} / 4=-c k / 4(1+c)$ (see Eq. (6)). With a constant $\eta_{2}$ Eq. (8) gives explicit RT and RM solutions [13]. For arbitrary $A$ and $\eta_{0}=\eta^{*}$ Eq. (5) reduces to

$$
\ddot{\theta}_{L}-g k_{L} A_{L} \theta_{L}=0
$$

where

$$
\begin{gathered}
k_{L} \equiv c(1+c)(1+A) k / 2(1+c+c A-A), \\
A_{L} \equiv 2 A /(1+c+c A-A),
\end{gathered}
$$

and

$$
\theta_{L} \equiv e^{\left(\eta-\eta_{0}\right) k_{L}}
$$


Therefore,

$$
\eta=\eta_{0}+\frac{1}{k_{L}} \ln \theta_{L}
$$

is the nonlinear solution. Since Eq. (10) has the same form as the linear equation, Eq. (1), we see that the nonlinear solution is essentially the logarithm of the linear solution. More explicitly,

$$
\eta^{\text {nonlinear }}=\eta_{0}+\frac{1}{k_{L}} \ln \eta^{\text {linear }}\left(1, \dot{\eta}_{0} k_{L} ; k_{L}, A_{L} ; g ; t\right)
$$

where $\eta^{\text {linear }}\left(\eta_{0}, \dot{\eta}_{0} ; k, A ; g ; t\right)$ is the solution to Eq. (1) with obvious notation.

In this model one uses the linear equation, Eq. (1), until $\eta$ reaches $\eta^{*}$, then switches to Eq. (10). For simplicity we have proposed [18] using Eq. (10) from the start for all $\eta_{0} \geq \eta^{*}$, even for $\eta_{0}>\left(\eta_{0}\right)_{\max }$ where the level 2 model fails, as mentioned above. An example later (Fig. 4) will compare these two models.

For the classical RT and RM cases the nonlinear solutions follow immediately from Eq. (13) using the linear solutions (2) and (3):

$$
\eta(t)=\eta_{0}+\frac{1}{k_{L}} \ln \left[\cosh \left(\gamma_{L} t\right)+\frac{\dot{\eta}_{0} k_{L}}{\gamma_{L}} \sinh \left(\gamma_{L} t\right)\right]
$$

and

$$
\eta(t)=\eta_{0}+\frac{1}{k_{L}} \ln \left(1+\dot{\eta}_{0} k_{L} t\right)
$$

respectively, where $\gamma_{L} \equiv \sqrt{g k_{L} A_{L}}$, and we have restored the sinh term in the RT expression. The asymptotic bubble velocities are

$$
\dot{\eta}_{\infty}=\gamma_{L} / k_{L}=\sqrt{g A_{L} / k_{L}}, \quad \mathrm{RT},
$$


and

$$
\dot{\eta}_{\infty}=1 / k_{L} t, \quad \mathrm{RM}
$$

Note that the nonlinear RM amplitude, Eq. (15), is the integral over

$$
\dot{\eta}(t)=\frac{\dot{\eta}_{0}}{1+\dot{\eta}_{0} / \dot{\eta}_{\infty}}=\frac{\dot{\eta}_{0}}{1+\dot{\eta}_{0} k_{L} t}
$$

given in [13]. This equation can be compared with other, substantially more complex expressions found in other models for the bubble velocity $\dot{\eta}[19,20]$.

Given its simplicity (compare Eqs. (5) and (10)) it is surprising how well this level 3 model performs in comparison with the level 2 model. We know of no example where the level 2 model does substantially better than this simple level 3 model. Add the advantage just mentioned for $\eta_{0}>\left(\eta_{0}\right)_{\max }$ and we see no reason to revert to the full equations Eq. (10) is equally, if not more, adequate. This is fortunate because the next and last model is based on it.

Level 4. The last and simplest model is obtained by applying a WKB-like approximation to Eq. (10): Define

$$
S \equiv \int_{0}^{t} \sqrt{g(t)} d t
$$

and cast Eq. (10) into the form

$$
\frac{d^{2} \theta_{L}}{d s^{2}}-k_{L} A_{L} \theta_{L}+\frac{1}{2 g^{2}} \frac{d g}{d t} \frac{d \theta_{L}}{d t}=0
$$

For $g(t)$ 's where the last term in the above equation can be neglected we obtain

$$
\theta_{L}=\cosh \left(s \sqrt{k_{L} A_{L}}\right)
$$

where we assumed $\dot{\theta}_{L}(0)=0$ and of course $\theta_{L}(0)=1$ by definition. Therefore, 


$$
\eta=\eta_{0}+\frac{1}{k_{L}} \ln \left[\cosh \left(s \sqrt{k_{L} A_{L}}\right)\right]
$$

is the nonlinear solution for "all" $g(t)$ and therefore called, quite appropriately, the scaling solution. This is the level 4 model. The quotation marks around "all" remind us that $g(t)>0$ and that $\dot{g} / \mathrm{g}^{2}$ must be small enough to justify dropping the last term in Eq. (20) (See Ref. [18]).

From Eq. (22) and the definition of $S$, Eq. (19), we obtain

$$
\dot{\eta}(t)=\sqrt{g} d \eta / d s=\sqrt{g A_{L} / k_{L}} \tanh \left(s \sqrt{k_{L} A_{L}}\right)
$$

for the bubble velocity. Asymptotically,

$$
\eta \rightarrow \eta_{\infty}=s \sqrt{A_{L} / k_{L}}=\sqrt{A_{L} / k_{L}} \int_{0}^{t} \sqrt{g} d t
$$

and

$$
\dot{\eta} \rightarrow \dot{\eta}_{\infty}=\sqrt{g(t) A_{L} / k_{L}}
$$

Needless to say, the scaling solution becomes exact for the standard RT case ( $g=$ const.) It fails, of course, for the RM case $(g=0)$, but an explicit nonlinear solution is known and was given in Eq. (15).

We conclude this section by recalling that our discussion has been limited to bubbles only. Spikes and their scaling will be taken up in Sec. IV. We hope it is clear how each level follows from the previous one. As promised, we evaluate levels 2 through 4 by comparing them with numerical simulations of gedanken experiments patterned after LEM experiments [5]. 


\section{GEDANKEN LEM EXPERIMENTS}

Fig. 1 displays three acceleration profiles which are very similar to the experimental $g(t)$ 's - See Fig. 2 in Ref. [5]. A fourth quasi-constant acceleration will be considered below. We apply these somewhat idealized acceleration profiles to the top and the bottom of the simulated LEM tank filled with hexane $\left(\rho=0.66 \mathrm{~g} / \mathrm{cm}^{3}\right)$ and a water/NaI solution ( $\rho=1.87 \mathrm{~g} / \mathrm{cm}^{3}$ ), for which $A \approx 0.48$. We use ideal equations of state with a high " $\gamma$ ”, the specific heat ratio, to reduce the effects of compressibility (CALE is a 2D compressible code but level 2 through 4 models assume constant $\rho_{A, B}$ ). The interface follows the prescribed acceleration but with some oscillations induced by sound waves See Ref. [18] and below.

The interface between the two fluids is initialized as a $\lambda=7.3 / 3 \mathrm{~cm}$ perturbation, i.e., three wavelengths across the 7.3-cm-wide tank. The height of the tank is $8.8 \mathrm{~cm}$. In some $A \approx 1$ calculations discussed in Sec. IV we needed to double this height to avoid spikes splashing against the roof of the tank. The initial amplitude is less than, equal to, or larger than $\eta^{*}=1 / 3 k=\lambda / 6 \pi=7.3 / 18 \pi \approx 0.13 \mathrm{~cm}$.

Fig. 2 compares the numerical simulations with the level 2 and 3 models for the three $g(t)$ 's shown in Fig. 1, starting with $\eta_{0}=0.065 \mathrm{~cm}$. It is practically impossible to differentiate between level 2 and level 3 models, and both come very close to the CALE simulation which, as mentioned earlier, we use as a measure of quality.

All three profiles in Fig.1 have one common element: Linearly increasing or decreasing acceleration. As shown in the Appendix, the solution in such a case is given by Airy functions. For the first leg where $g=\dot{g} t$ and starts with $\dot{\eta}_{0}=0$ we can write 


$$
\eta(t)=\frac{\pi \eta_{0}}{3^{1 / 3} \Gamma(1 / 3)}[\operatorname{Bi}(z)+\sqrt{3} A i(z)]
$$

where $\mathrm{z} \equiv \mathrm{t} / \mathrm{T}$ with $\mathrm{T} \equiv|\dot{\mathrm{g} k A}|^{-1 / 3}$, assuming $\dot{\mathrm{g} A}>0$. For $\dot{\mathrm{g} A}<0, \mathrm{z} \equiv-\mathrm{t} / \mathrm{T}$.

Let us compare with the classical RT case $g=$ const. given by Eq. (2):

$$
\eta(t)=\frac{\eta_{0}}{2}\left[e^{\gamma t}+e^{-\gamma t}\right]
$$

where $\gamma \equiv|g k A|^{1 / 2}$. The Bi term in Eq. (26) corresponds to $e^{\gamma t}$ in Eq. (27), both increasing for large $t$, while the $A i$ term mirrors $e^{-\varkappa}$, both decreasing for large $t$. For a stable acceleration $(\mathrm{gA}<0) \cosh \rightarrow \cos$, i.e., $e^{ \pm \lambda t} \rightarrow e^{ \pm i \lambda t}$, both terms in Eq. (27) oscillate, just as $A i$ and $B i$ do for a negative argument.

We saw how the simple rule of Eq. (13) transformed Lord Rayleigh's linear solution, Eq. (2), and Richtmyer's linear solution, Eq. (3), into our nonlinear solutions Eqs. (14) and (15), for the classical RT and RM cases respectively. Similarly, for the case $g=\dot{\mathrm{g} t}$ the nonlinear solution follows immediately from Eq. (26):

$$
\eta(t)=\eta_{0}+\frac{1}{k_{L}} \ln \left\{\frac{\pi}{3^{1 / 3} \Gamma(1 / 3)}\left[\operatorname{Bi}\left(z_{L}\right)+\sqrt{3} A i\left(z_{L}\right)\right]\right\},
$$

where $\mathrm{z}_{\mathrm{L}} \equiv \mathrm{t} / \mathrm{T}_{\mathrm{L}}$ with $\mathrm{T}_{\mathrm{L}} \equiv\left|\dot{g} k_{L} A_{L}\right|^{-1 / 3}$.

As mentioned in the Introduction, "constant acceleration" LEM experiments maintained a constant acceleration only after a linear climb from $\mathrm{g}=0$ to $\mathrm{g}=\mathrm{g}_{\max }=\dot{g} \tau$ at $\mathrm{t}=\tau$. During the climb Eq. (26) or, more probably Eq. (28) describes the bubble amplitude. After the tank reaches $g_{\max }$ and maintains a constant 
acceleration Eq. (14) describes the evolution. If, instead of maintaining a constant $g$ the tank is brought back to rest with a linearly decreasing $g$, as in Figs. 1a-1c, then Eq. (28), with a straightforward generalization to include $\dot{\eta}_{0}$, also describes this phase. The case of an impulse, as in Fig. 1c, is treated explicitly in the Appendix.

Any acceleration profile can be broken down into a series of piecewise linear sections. Any linear $g(t)=g_{0}+\dot{g} t$ can be cast into Airy's equation and therefore $\eta(t)$ in each interval is given by a linear combination of Airy functions.

We now turn to a quasi-constant acceleration:

$$
g(t)=g_{\infty}\left(1-e^{-t / T}\right)
$$

varying smoothly from 0 to $g_{\infty}$ over a timescale $T$. It is plotted, using arbitrary units, in Fig. 3. CALE results for $g_{\infty}=35 g_{E}$ and $T=1.2 \mathrm{~ms}$, are also shown in Fig. 3, along with the analytic solution

$$
\eta(t)=\eta_{0}+\frac{1}{k_{L}} \ln \left[\left(c_{+} \sin z_{L}+c_{-} \cos z_{L}\right) / \sqrt{z_{L}}\right] .
$$

We have taken $\eta_{0}=\eta^{*}=0.13 \mathrm{~cm}, k_{L}=3(1+A) k /(3+A) \approx 1.28 \mathrm{k} \approx 3.3 \mathrm{~cm}^{-1}$, and $c_{ \pm} \equiv\left(\cos \frac{1}{2} \pm \sin \frac{1}{2}\right) / \sqrt{2}$ giving $c_{+} \approx 0.96$ and $c_{-} \approx 0.28$. The variable $z_{L}$ in Eq.

(30) is defined as $z_{L}=\frac{1}{2} e^{-t / 2 T_{L}}$ where $T_{L} \equiv 1 / 4 \sqrt{g_{\infty} k_{L} A_{L}}$. The derivation of Eq. (30) is also left to the Appendix. As expected, $\dot{\eta}$ asymptotes to the classical result $\dot{\eta} \rightarrow \sqrt{g_{\infty} A_{L} / k_{L}}$ for constant acceleration (See Eq. (16)). 
Let us use this example of quasi-constant acceleration to illustrate two points we have briefly discussed in previous communications: (1) Failure of Eq. (5) for $\eta_{0}>\left(\eta_{0}\right)_{\max }$, and (2) zitterbewegung [17,18].

We ran the same problem on CALE starting with $\eta_{0}=4 \eta^{*} \approx 0.52 \mathrm{~cm}$ which, using Eq. (9), is larger than $\left(\eta_{0}\right)_{\max } \approx 0.48 \mathrm{~cm}$. The results are shown in Fig. 4. The level 2 model clearly fails. The level 3 model, Eq. (10) for all $\eta_{0} \geq \eta^{*}$, gives a reasonable answer. Since Eq. (30) is this level 3 solution to Eq. (10), all we had to do was set $\eta_{0}$ to the appropriate value. In this model, which we prefer, increasing $\eta_{0}$ merely shifts the curve up by a constant.

Turning to zitterbewegung, here meaning rapid oscillations of $g(t)$ around an average value, we borrowed the term from Quantum Mechanics because Eq. (10), like Eq. (1), has the same form as the Schrödinger equation: We solve an initial value problem in time instead of an eigenvalue problem in space. As mentioned in [18], we first became aware of zitterbewegung when we compared the acceleration of the interface with the idealized acceleration imposed at the top and the bottom of the simulated tank in CALE. Sound waves in the compressible code induce small oscillations. In Fig. 5 we display the idealized $g(t)$, Eq. (29), and the code-calculated acceleration in thin dashed lines, showing these oscillations. Increasing (decreasing) the compressibility of the fluids by decreasing (increasing) the specific heat ratio " $\gamma$ ” increased (decreased) the oscillations but made practically no difference to the resulting $\eta(t)$. Similarly, when we used the code-calculated rapidly oscillating acceleration as $g(t)$ in Eq. (10), we obtained 
essentially the same result. This led us to impose deliberately large oscillations on the ideal $g(t)$ to amplify the effect:

$$
g(t)=g_{\infty}\left(1-e^{-t / T}\right)(1+\varepsilon \cos \omega t)
$$

also displayed in Fig. 5 with $\varepsilon=1$, taking $\omega=2 \pi / 10 \mathrm{~ms}^{-1}$. This caused $g(t)$ to vary by $\pm 100 \%$, from 0 to $70 g_{E}$, instead of the average $35 g_{E}$. To focus on the effect of such an oscillation let us define

$$
\delta(t ; \varepsilon) \equiv \frac{\eta(t ; 0)-\eta(t ; \varepsilon)}{\eta(t ; 0)}
$$

as the deviation in $\eta$ without ( $\varepsilon=0$ ) and with ( $\varepsilon \neq 0$ ) oscillations, keeping everything else the same (we suppress the dependence on $\omega$ ). Fig. 6 shows $\delta(t ; 1)$ as calculated by CALE and by Eq. (10). The $100 \%$ difference in $g(t)$ translates to no more than $~ 13 \%$ difference in $\eta(t)$.

The rest of this section is devoted to what may be called "double-shock" or "doubleimpulse” experiments. No physical experiments of this type have been performed. The concept originated from shock tube experiments in which the interface, after seeing the first incident shock, is shocked a second time by the reflected shock, i.e., the incident shock passes thru the interface, hits the endwall of the tube, reflects, and returns to reshock the (already moving) interface. Since all RM experiments in shock tubes have an endwall, they all have a second (and subsequently $3^{\text {rd }}, 4^{\text {th }}$, etc.) shock. For examples see Refs. [21-23]. A systematic numerical and analytic treatment can be found in [17].

If we call the impulse in Fig 1c the first "shock", a second shock would be an impulse just like it in the same or, more interestingly, in the opposite direction, an example of which is shown in Fig. 7. These are two equal and opposite impulses, so the interface 
comes to rest immediately after the second impulse, i.e., at $30 \mathrm{~ms}$. In this case, with the impulses having $\left|g_{\max }\right|=70 g_{E}$, the interface moves $6.2 \mathrm{~cm}$ and then stops.

Since this involves positive as well as negative values of $g(t)$ the reader may anticipate difficulty with the models and indeed all models fail except, of course, the level 1 model. This is shown in Fig. 8 for the "bubble" amplitude calculated by CALE, showing the phase reversal, i.e., the interchange of bubbles and spikes, and the level 2 calculation which fails at $30 \mathrm{~ms}$. The level 3 model also fails at the same location. As for the level 4 (scaling) model, one cannot even define a real $s(t)$ for negative $g(t)$. A similar failure was reported in [17].

After $30 \mathrm{~ms}$, when $g=0$, it is possible to follow the new bubble amplitude which grows logarithmically according to our nonlinear RM formula, Eq. (15), once $\eta$ (30 ms) and $\dot{\eta}$ (30 ms) are known. Snapshots of the CALE simulation are shown in Fig. 9, from which $\eta$ (30 ms) $\approx 0$. A similar procedure, including the spike, will be described in the next section. As expected, given $g=0$ and the correct $\eta_{0}$ and $\dot{\eta}_{0}$ Eq. (15) agrees quite well with CALE simulations, as previously reported for single shock experiments.

Such double-impulse experiments have a great advantage for diagnostics: The tank comes to rest while perturbations evolve, similar to what happens in shock tubes where the interface slows down substantially upon reshock (but does not quite come to rest).

Obviously, all tanks must come to rest at the end of an experiment $\left(\int_{0}^{t} g d t=0\right)$; the proposed double-impulse experiment brings it to rest in the middle of the experiment and 
mixing can go on "forever" (we have neglected Earth's gravity which will probably induce very slow demixing).

Note that $\eta(t)$ overshoots - it changes phase in Fig. 9. Clearly, a somewhat weaker second impulse can "freeze-out" $\eta(t)$, again borrowing shock-tube terminology [24]. An example of such a $g(t)$ is shown in Fig. 10, and the corresponding $\eta$ 's, starting with $\eta_{0}=0.065 \mathrm{~cm}$, are shown in Fig. 11. Three curves are plotted in Fig. 11: 1) A calculation by CALE which shows complete freeze-out; 2) a calculation with Eq. (5) indicating slow increase; and 3) a calculation with Eq. (10) indicating slow decrease. Only by going to such "critical” phenomena do we see a difference between the level 2 and the level 3 models; for most practical purposes there is no difference. The fourth fast growing curve in Fig. 11 shows how $\eta(t)$ would grow without the reshock. Only the CALE calculation is shown for that no-reshock case because Eqs. (5) and (10) both get this evolution correctly as it is simply a "single-shock" problem like Fig. 1c.

The strategy for inducing freeze-out is fairly simple. First, it is necessary to have $g=0$ so $\dot{\eta}$ does not change (See Eq. (1) or (10)). This is necessary but not sufficient: Not only $\dot{\eta}$ must not change, it must be zero, by definition of freeze-out. The strategy is therefore to turn off the acceleration the precise moment $\dot{\eta}$ reaches 0 . The acceleration profile prior to this freeze-out time is almost arbitrary - any $g(t)$ which gives $\dot{\eta}=0$ at some time will do. It may even be possible to stop the bulk motion at $t_{\text {freeze-out }}$ which would add the constraint $\int_{0}^{t_{\text {freeze-out }}} g(t) d t=0$, a rather weak requirement. 
We have discussed these issues in a two-shock system and in the linear regime [24]. Freeze-out is possible in the nonlinear regime also [25]. We have pointed out that in such a system it is not possible to stop the interface and freeze-out $\eta$ simultaneously: Stopping the tank requires an equal and opposite reshock, but since $\eta$ grows between shock and reshock the effect of the reshock is amplified and hence it causes a phase reversal instead of freeze-out - it is "too strong". This is a well known occurrence in all shock tubes with a solid endwall. Recently, however, a new technique for reducing the reshock was incorporated in a shock tube using partial shock absorbers [26]. Although this method was used to investigate the RM turbulent mix [26], reducing the reshock is exactly what is required to freeze-out a perturbation with a judiciously chosen wavelength and reshock time [24]. We hope future experiments will pursue the detection of this phenomenon.

\section{SCALING OF BUBBLES AND SPIKES}

In all our graphs we have plotted $\eta$, the bubble amplitude, as a function of time, naturally. Induced by different acceleration histories $g(t)$, they have different time dependences - compare figures $2 \mathrm{a}, 2 \mathrm{~b}, 2 \mathrm{c}$, and 3 . The idea of scaling is that there may be a variable, in this case $S$ as defined by Eq. (19), which collapses all the different time evolutions into one universal curve. In our model that would be Eq. (22). To test this hypothesis we plot in Fig. 12 all four curves of $\eta$ as functions of $s$ - these are the solutions to Eq. (10) starting with $\eta_{0}=\eta^{*}=1 / 3 k$ using the four acceleration histories of Fig. 1a, 1b, 1c, and the quasi-constant $g(t)$ of Eq. (29), labeled A, B, C, D for brevity.

For each case we computed $\eta(t)$ vs $t, s(t)$ vs $t$, and display $\eta$ vs $s, t$ being the 
parametric variable. Curve E is Eq. (22). We see that all $g(t)$ 's scale, except for the impulsive case C because $g=0$ after $s \approx 5 \mathrm{~cm}^{1 / 2}$. Until that "time" C also scales.

As mentioned in [18], the parameter $S$ was suggested first by Read [4] based on his rocket-rig experiments. Subsequently, it was confirmed by Dimonte and Schneider [5], all for the turbulent bubble mixing width $h^{b}$, finding $h^{b} \approx \alpha^{b} A s^{2}$ with $\alpha^{b} \approx 0.05-0.07$ [4,5,6]. For the single-wavelength bubble amplitude applying a WKB-like approximation to Eq. (10) we obtained Eq. (22), a different scaling expression, predicting $\eta \rightarrow s \sqrt{A_{L} / k_{L}}$ asymptotically. We believe Eq. (22) is the simplest level 4 model - an explicit solution for "all” $g(t)$. It is of course less accurate than the level 3 model, Eq. (10), which described case C even after $g=0$, giving Eq. (15).

Let us now use CALE to test the scaling hypothesis with three different accelerations, again labeled A, B, and C for brevity. A is a constant acceleration of $70 g_{E}$; B is a cubic function of $t$,

$$
g=g_{3} t^{3}
$$

with $g_{3}=0.06 g_{E} / \mathrm{ms}^{3}$, so that by $20 \mathrm{~ms} g=480 g_{E}$. C is again an impulsive acceleration reaching a peak of $500 \mathrm{~g}_{\mathrm{E}}$ by $\tau=1 \mathrm{~ms}$ and returning to zero after another millisecond. In this case a jump velocity $\Delta \mathrm{v}=\int \mathrm{gdt}=g_{\max } \tau=0.49 \mathrm{~cm} / \mathrm{ms}$ is achieved by 2 ms and remains constant thereafter. These three acceleration profiles are plotted in Fig. 13a. The corresponding displacements $\Delta x(t)$ are shown in Fig. 13b, and the corresponding $s(t)$ in Fig. 13c. 
The bubble and spike amplitudes (the latter negative in our convention) as calculated by CALE are shown in Fig. 14a as functions of time. All start with $\left|\eta_{0}\right|=\eta^{*}=0.13 \mathrm{~cm}$. Since they all have different $g(t)^{\prime} \mathrm{s}$, it is not surprising to see different evolutions with time. Let us consider $\Delta x(t)$ and $s(t)$ as possible candidates for scaling. We plot the same data as functions of $\Delta x(t)$ in Fig. 14b, and as functions of $s(t)$ in Fig. 14c.

Fig. 14c confirms the expected scaling of bubbles with $s$ : Curves A and B are very close to each other in that figure. So is curve C until, of course, $g=0$. The spikes do not scale with $S$, as reported earlier [18].

Fig. 14b, however, shows that spikes scale with $\Delta x$ : Spikes A and B are very close to each other when plotted as functions of $\Delta x$. So is C until $g=0$, i.e. $t=2 \tau=2 \mathrm{~ms}$, by which time $\Delta x_{C}(2 m s)=0.49 \mathrm{~cm}$ only. As far as we know there has been no earlier determination of scaling of spikes. Dimonte and Schneider considered scaling of bubbles with $\Delta x$ and abandoned it in favor of $s$ [5]. While bubbles do scale with $s$, spikes apparently scale with $\Delta x$.

It is well known that bubbles and spikes differ in shape and in time-evolution at moderate to high Atwood numbers. All our calculations so far were limited to $A \approx 0.48$. At low $A$ bubbles and spikes behave similarly and therefore both must scale, if at all, with the same variable. We carried out a low- $A$ simulation $(A \approx 0.17)$ but could not determine whether $s(t)$ or $\Delta x(t)$ was the better scaling variable: Both were equally good (or poor) to within $10-20 \%$.

At high Atwood numbers bubbles scale with $s(t)$ while spikes scale with $\Delta x(t)$. We repeated our A, B, and C accelerations after replacing the low-density hexane ( $\rho=0.66$ 
$\left.\mathrm{g} / \mathrm{cm}^{3}\right)$ with air $\left(\rho=0.0012 \mathrm{~g} / \mathrm{cm}^{3}\right)$, so that $A \approx 1$. The resulting amplitudes are displayed in Fig. 15. As expected, there is now much more contrast between bubbles and spikes, the latter growing very large. To accommodate the late-time spikes $(\sim 9 \mathrm{~cm})$ and bubbles $(\sim 3 \mathrm{~cm})$ we doubled the height of the tank. Again, the evolution of $\eta^{\text {bubble }}(t)$ and $\eta^{\text {spike }}(t)$ as functions of $t$ are different for the different $g(t)$ 's, but spikes prefer scaling with $\Delta x$ (Fig. 15b) while bubbles continue to scale with $s$ - See Fig. 15c.

The following argument motivates our claim that at moderate to high Atwood numbers spikes scale with $\Delta x$. We have seen that for bubbles with $\eta>0$ the curvature (Eq. (6)) generally asymptotes to a constant and can even be kept absolutely constant with the choice $\eta_{0}=\eta^{*}$. In contrast, for spikes with $\eta<0$ the curvature cannot be kept constant and, at late times, $\eta_{2}(t) \sim e^{-\eta k} \rightarrow \infty$ [14]. Now, with a large $\eta_{2}(t)$ only the first and last terms in Eq. (8) contribute:

$$
\eta_{2}(\ddot{\eta}+g)=0
$$

from which

$$
\eta=-\int\left[\int g d t\right] d t=-\Delta x
$$

confirming the scaling of spikes with $\Delta x$.

This single-fluid $A=1$ case remains the most advanced nonlinear model - The governing equation is Eq. (8) with the curvature $\eta_{2}(t)$ given by Eq. (6). Layzer considered $\eta_{0}=0$ and bubbles only [12]. We considered arbitrary $\eta_{0}$, noted the simplification for $\eta_{0}=\eta^{*}$, and gave explicit solutions for the RM case with arbitrary $\eta_{0}$, again for bubbles only [13]. Subsequently, Zhang proposed using the same equations 
with negative $\eta_{0}$ and $\dot{\eta}_{0}$ for spikes [14]. It follows that the analytic solutions we had derived for $\eta_{0}<\eta^{*}$ apply to spikes also, as noted recently [10].

The exact RM solution to Eq. (8) is Eq. (11) in Ref. [13], valid for $g=0$ and $\eta_{0}<\eta^{*}=1 / 3 k$ (We are considering 2D; for 3D use Eq. (14) in Ref. [13].) Let us set $\eta_{0}=0$ in that equation which then becomes

$$
\dot{\eta}_{0} k t=\frac{2 \sqrt{2}}{3 \sqrt{3}} Y-\frac{2}{3}+\frac{1}{3 \sqrt{3}} \ln \left[\frac{(\sqrt{3}+1)(\sqrt{2} Y-1)}{(\sqrt{3}-1)(\sqrt{2} Y+1)}\right]
$$

where

$$
Y^{2} \equiv e^{3 \eta k}+1 / 2
$$

As a check, at $t=0 \quad Y=\sqrt{3 / 2}$ and the left- and right-hand-sides of Eq. (36) vanish.

There are two reasons why Eq. (36) is interesting. First, Layzer obtained a first integral of his equation giving the bubble velocity $\dot{\eta}^{\text {bubble }}$ by considering $\eta_{0}=0$ and $g=$ const. A second integral needed to obtain $\eta^{\text {bubble }}$ could be done only by numerical quadrature [12]. Had he considered $g=0$, he would have obtained this second integral analytically: The above Eq. (36). Of course, Layzer’s paper [12] was published 5 years before Richtmyer's [2] so the RM instability was not known at the time to justify $g=0$ (However, we note that Richtmyer's original classified report was written in July 1954 and was probably known to Layzer).

The second reason why Eq. (36) is interesting is that since it is derived by setting $\eta_{0}=0$ in Eq. (11) of Ref. [13] it applies, as is, to both bubbles and spikes. For bubbles, $\dot{\eta}_{0}>0$. For spikes, $\dot{\eta}_{0}<0$, following Zhang's suggestion for spikes [14]. 
Eq. (36) is compared with a CALE simulation in Fig 16. The CALE problem, starting with $\eta_{0}=0.13 \mathrm{~cm}$, is the same as the impulsive problem C considered in Figs. 13-15, except the impulse is negative, i.e., $g$ goes from 0 to $-500 g_{E}$ by $t=\tau=1 \mathrm{~ms}$, and returns to 0 by $2 \tau=2 \mathrm{~ms}$. As expected, the perturbation changes phase going through zero at $t \approx 2 \mathrm{~ms}$ (the bubble at $2.1 \mathrm{~ms}$, the spike at $1.8 \mathrm{~ms}$ ), because the shock is now proceeding from the high density to the low density fluid - in other words, the tank is jolted up instead of down. We apply Eq. (36) starting at $t=2 \mathrm{~ms}$.

To estimate $\dot{\eta}_{0} \equiv \dot{\eta}(2 \tau)$ in Eq. (36) we use the approximate expression

$$
\dot{\eta}(2 \tau)=\eta_{0} \Delta \mathrm{v} k A(1+7 \Delta \mathrm{v} k A \tau / 30+\ldots)
$$

given in [18] and derived in the Appendix (see Eq. (A30b)). The well-known leading term, $\eta_{0} \Delta v k A$, was given by Richtmyer and applies for $g \rightarrow \infty$ with $\tau \rightarrow 0$. The second term above reflects the finite width of the pulse and comes from expanding the exact expression, Eq. (A26b), which involves Airy functions. We note that Eqs. (A30a,b) are valid for both $\Delta \mathrm{v} A>0$ and $\Delta \mathrm{v} A<0$.

Setting $\eta_{0}=0.13, \Delta \mathrm{v} A=-0.49 \mathrm{~cm} / \mathrm{ms}, k=6 \pi / 7.3 \approx 2.58 \mathrm{~cm}^{-1}$, and $\tau=1$ ms, Eq. (38) gives $|\dot{\eta}(2 \tau)| \approx 0.12 \mathrm{~cm} / \mathrm{ms}$. The solid curves in Fig. 16 are obtained from Eq. (36) after shifting $t$ by $2 \mathrm{~ms}$ and using $\pm 0.12 \mathrm{~cm} / \mathrm{ms}$ for $\dot{\eta}(2 \tau)$. We see that starting from $\eta_{2 \tau}=0$ the same equation, Eq. (36), with a positive (negative) $\dot{\eta}_{2 \tau}$ gives the bubble (spike) amplitude and agrees well with the CALE simulation. The insets in Fig. 16 show the beginning ( $t=0)$ and ending ( $t=24 \mathrm{~ms}$ ) configurations of the heavy fluid. Clearly, the spikes are much longer than the bubbles. A similar configuration is reached with constant $g$ (Fig. 4 in Ref. [10]). 
The spike evolution has several applications as it is similar to that of shaped charges [27], ejecta [28], and of course jets [29].

Asymptotic bubble and spike amplitudes can be obtained from Eq. (36) using $Y \rightarrow e^{3 \eta k / 2}$ for bubbles $\left(\eta^{b}>0\right)$ and $Y \rightarrow\left(1+e^{3 \eta k}\right) / 2$ for spikes $\left(\eta^{s}<0\right)$ :

$$
\eta^{b} \rightarrow \frac{2}{3 k} \ln \left(\frac{3 \sqrt{3} \dot{\eta}_{0} k t}{2 \sqrt{2}}\right)
$$

and

$$
\eta^{s} \rightarrow \sqrt{3} \dot{\eta}_{0} t
$$

Using $t=22 \mathrm{~ms}, \dot{\eta}_{0}=0.12 \mathrm{~cm} / \mathrm{ms}$, and $k=2.58 \mathrm{~cm}^{-1}$ in Eqs. (39a,b) we find $\eta^{b} \approx 0.65 \mathrm{~cm}$ and $\eta^{s} \approx-4.6 \mathrm{~cm}$ at $24 \mathrm{~ms}$, in agreement with CALE, as seen in Fig. 16. The asymptotic bubble velocity $\dot{\eta}^{b} \rightarrow 2 / 3 k t$ from Eq. (39a) agrees with $\dot{\eta}_{\infty}=2 / 3 k t$ given in [13], and the asymptotic spike velocity $\dot{\eta}^{s} \rightarrow \sqrt{3} \dot{\eta}_{0}$ from Eq. (39b) agrees with the $\eta_{0}=0$ version of the equation

$$
\left(\frac{\dot{\eta}^{s}}{\dot{\eta}_{0}^{s}}\right)_{\eta^{s} \rightarrow-\infty}^{2}=\frac{1-\eta_{0} k}{1 /(1+c)-\eta_{0} k}=\frac{1+\left|\eta_{0} k\right|}{1 /(1+c)+\left|\eta_{0} k\right|}
$$

given in [10]. Note that the $\sqrt{3}$ in Eq. (39b) is the largest factor one can have - it is replaced by $\sqrt{2}$ for 3D spikes. It is interesting that it gets even smaller if the initial amplitude $\eta_{0}$ does not vanish and reduces from $\sqrt{3}$ to $\sqrt{1}$ for large $\left|\eta_{0} k\right|$. It may be counterintuitive that large initial amplitudes lead to smaller $\dot{\eta}_{\infty} / \dot{\eta}_{0}$, as in Eq. (40), but one must remember that $\dot{\eta}_{0}$ itself is an increasing function of $\eta_{0}$ (linearly in the linear 
regime) so that shaped charges or ejecta or jets with larger initial amplitudes will indeed have larger asymptotic velocities. We ran CALE simulations with $\eta_{0}=0.065,0.13$ (shown in Fig. 16), 0.26, and $0.52 \mathrm{~cm}$, obtaining $\left|\dot{\eta}_{\infty}^{s}\right|=0.09,0.19,0.38$, and $0.68 \mathrm{~cm} / \mathrm{ms}$ respectively, showing almost linear dependence of $\dot{\eta}_{\infty}^{s}$ on $\eta_{0}$. Of course to get the actual (laboratory) speed of the tip of the jets one must add the tank velocity, $0.49 \mathrm{~cm} / \mathrm{ms}$, to $\dot{\eta}_{\infty}^{s}$. For example, for the case shown in Fig. 16 the laboratory speed of the jet is $0.19+0.49=0.68 \mathrm{~cm} / \mathrm{ms}$.

What happens if we reshock the tank and bring it to rest? As expected, the bubble and spike grow faster than the single-shock case, as shown in Fig. 17 where, starting at 10 ms, we sent an equal and opposite impulse lasting until $12 \mathrm{~ms}$, bringing the tank to rest $4.9 \mathrm{~cm}$ above its original position. By $24 \mathrm{~ms}$ the total extent of the jet, $\left|\eta^{b}\right|+\left|\eta^{s}\right|$, is more than twice the single-shock case: $10.6 \mathrm{~cm}$ here vs $4.8 \mathrm{~cm}$ in Fig. 16. Clearly, the spike is by far the dominant component, making up $9.5 \mathrm{~cm}$ of the $10.6 \mathrm{~cm}$ - see Fig. 17 . Its motion is actually quite simple to describe: Since the spike has a small cross section $\left(1 / \eta_{2}^{s} \rightarrow 0\right)$ it is not affected much at all by the reshock and continues as before, i.e. at $\sim 0.68 \mathrm{~cm} / \mathrm{ms}$ in the laboratory, and indeed from Fig. $17 \dot{\eta}^{s} \approx-0.68 \mathrm{~cm} / \mathrm{ms}$ after 12 ms. The effect of the reshock is simply to stop the bulk motion of the tank without affecting the spike; this is the reason for the apparent increase in $\left|\dot{\eta}^{\text {s}}\right|$ seen in Fig. 17 at 12 ms. Fig. 18 compares the shock and reshock cases side by side at 24 ms: In the first, shock-only case (left figure) the tank is moving up at $0.49 \mathrm{~cm} / \mathrm{ms}$ and $\left|\dot{\eta}^{s}\right|$ is apparently small; in the second case with reshock (right figure) the tank is at rest and $\left|\dot{\eta}^{\text {s }}\right|$ is large. In 
both cases the jet locations and speeds in the laboratory are approximately the same, 15 $\mathrm{cm}$ and $\sim 0.68 \mathrm{~cm} / \mathrm{ms}$, respectively.

\section{CONCLUDING REMARKS}

Layzer's approach can be summarized as using a simple potential in the nonlinear Bernoulli equation. Despite its known failures [10] it is a very powerful technique yielding explicit, analytic expressions valid mostly for the bubble amplitude and, as proposed by Zhang [14], for the spike when $A=1$. Probably the best illustration of the model is Eq. (36), valid for both bubbles and spikes. As we reasoned, Layzer could have derived it had he set $g=0$ in his equations because he considered $\eta_{0}=0$. For arbitrary $\eta_{0}$ one can revert to Eq. (11) in [13], using a positive (negative) $\eta_{0}$ for bubbles (spikes).

Goncharov's extension of the model to arbitrary $A$ [15] works very well for bubbles, but fails for spikes [10]. We believe this signals a deficiency in that extension and further work is needed. The model has been criticized for using a potential $\phi \sim y$ whose derivative (and hence the light-fluid-velocity) does not vanish at infinity [30], but we do not believe this to be essential - As Goncharov asserted, the potential is to be used near the interface $y \approx 0$ only and not at $y \rightarrow \infty$. What we have found attractive in Goncharov's extension is that it allows a simple transformation to convert our earlier $A=1$ bubble results to arbitrary $A$. It remains to be seen if a more advanced model for arbitrary A will continue to provide simple explicit solutions like Eqs. (14) and (15) for RT and RM instabilities.

Similarly, the scaling of bubbles with $s$ and the scaling of spikes with $\Delta x$ deserve more scrutiny. Experimentally, scaling has been addressed only for the turbulent mixing 
width and only for the bubble width $h^{b}$ [4,5]. We hope other simulations and experiments with single-scale perturbations will throw light on these issues by considering a variety of acceleration profiles $g(t)$. Even more useful will be the study of any scaling in the turbulent spike width $h^{s}$. Turbulence, being a 3D phenomenon requiring intensive computational capabilities, we leave for the future.

This work was performed under the auspices of the U. S. Department of Energy by Lawrence Livermore National Laboratory under Contract DE-AC52-07NA27344.

\section{APPENDIX}

In this Appendix we present solutions to Eq. (1) or Eq. (10) for various $g(t)$ 's . As we have repeatedly pointed out, both equations have the same mathematical structure and are similar to Schrödinger's equation. Yet another analogy is the "harmonic oscillator with a time-dependent spring constant $k(t)$," i.e., $\ddot{X}+\frac{k(t)}{m} X=0, X$ being the position and $m$ the mass of the load attached to the spring. Despite the appearance of such an equation in many fields of physics and finance, a general solution valid for arbitrary $k(t)(g(t)$ in our case) is not known. The results for the RT ( $g=$ const.) and $\mathrm{RM}(g=\Delta \mathrm{v} \delta(t))$ cases were given in the main body of this paper and will not be repeated.

1) $g(t)=g_{\infty} \tanh ^{2}(t / T)$. For simplicity of notation we solve Eq. (1). Define $y \equiv \cosh ^{2}(t / T)$ and substitute in Eq. (1) to obtain 


$$
y(1-y) \frac{d^{2} \eta}{d y^{2}}+\left(\frac{1}{2}-y\right) \frac{d \eta}{d y}-g_{\infty} \frac{k A T^{2}}{4}\left(\frac{1}{y}-1\right) \eta=0 .
$$

Now define $\eta \equiv y^{v} \sigma(y)$ where $v(v-1 / 2) \equiv g_{\infty} k A T^{2} / 4$ and write the above equation as

$$
y(1-y) \frac{d^{2} \sigma}{d y^{2}}+\left(2 v+\frac{1}{2}-(1+2 v) y\right) \frac{d \sigma}{d y}-\frac{v}{2} \sigma=0
$$

This being a hypergeometric equation, its solution is a linear combination of hypergeometric functions with the coefficients determined by the initial conditions $\eta_{0}$ and $\dot{\eta}_{0}$. The reader will probably recognize that we have duplicated a QM problem, translating space $x$ in the potential $V(x)$ to time $t$ in the acceleration $g(t)$.

Of course it is simpler to solve Eq. (1) numerically than look up tables for hypergeometric functions. Even simpler is the scaling solution, Eq. (22), with

$$
s(t)=T \sqrt{g_{\infty}} \ln (\cosh (t / T)) .
$$

We have verified that this is an extremely good approximation to the numerical solution.

2) $g(t)=g_{\infty}\left(1-e^{-t / T}\right)$. This was used as an example in Figs. 3 thru 6. Define

$$
\mathrm{z} \equiv n e^{-t / 2 T}
$$

where $n \equiv 2 \gamma T \equiv 2 T \sqrt{g_{\infty} k A}$, not necessarily an integer. Substituting in Eq. (1) we get

$$
\frac{d^{2} \eta}{d z^{2}}+\frac{1}{z} \frac{d \eta}{d z}+\left(1-\frac{n^{2}}{z^{2}}\right) \eta=0
$$


where $\eta=\eta(z)$. The domain $0 \leq t<\infty$ is mapped onto $n \geq z>0$ with $\eta_{0} \equiv \eta(t=0)=\eta(z=n)$. We shall take $\dot{\eta}_{0} \sim(d \eta / d z)_{z=n}=0$. The solution to Eq (A5) is

$$
\eta(t)=c_{1} J_{n}(z)+c_{2} J_{-n}(z)
$$

where $J_{ \pm n}$ are Bessel functions of the first kind and $c_{1,2}$ must be determined, as usual, by the initial conditions $\eta_{0}$ and $\dot{\eta}_{0}$. The problem is much simplified for the case $n=1 / 2$ as the Bessel functions reduce to $\sin z / \sqrt{z}$ and $\cos z / \sqrt{z}$. Henceforth we take $n=1 / 2$, i.e., $T=1 / 4 \gamma=1 / 4 \sqrt{g_{\infty} k A}$. Then

$$
\eta(t)=\eta_{0}\left(c_{+} \sin z+c_{-} \cos z\right) / \sqrt{z}
$$

where $c_{+}=\left(\cos \frac{1}{2}+\sin \frac{1}{2}\right) / \sqrt{2} \approx 0.96 \quad$ and $\quad c_{-}=\left(\cos \frac{1}{2}-\sin \frac{1}{2}\right) / \sqrt{2} \approx 0.28$, satisfying $\eta(0)=\eta(\mathrm{z}=1 / 2)=\eta_{0}$ and $\dot{\eta}_{0}=0$.

It is interesting that asymptotically, meaning $t \rightarrow \infty$ or $z \rightarrow 0$, $\eta \rightarrow \eta_{0} c_{-} / \sqrt{z}=\eta_{0} c_{-}\left(\sqrt{2} e^{t / 4 T}\right)=\eta_{0}(\cos (1 / 2)-\sin (1 / 2)) e^{\gamma t} \approx 0.4 \eta_{0} e^{\gamma t}$, compared with the solution to the standard RT linear problem, $\eta=\eta_{0} \cosh (\not t) \rightarrow 0.5 \eta_{0} e^{\varkappa}$

At late times the amplitude must surely be nonlinear so, instead of Eq. (A7) one should use

$$
\eta(t)=\eta_{0}+\frac{1}{k_{L}} \ln \left[\left(c_{+} \sin z_{L}+c_{-} \cos z_{L}\right) / \sqrt{z_{L}}\right.
$$


where $z_{L} \equiv n e^{-t / 2 T_{L}}=\frac{1}{2} e^{-2 \gamma_{L} t}$ with $\gamma_{L} \equiv \sqrt{g_{\infty} k_{L} A_{L}}$. Asymptotically, $\eta$ now grows only linearly with time with the well known asymptotic bubble velocity $\dot{\eta}_{\infty}=\gamma_{L} / k_{L}=\sqrt{g_{\infty} A_{L} / k_{L}}$.

What about the scaling formula, Eq. (22)? For this $g(t)$ one finds

$$
s(t)=T \sqrt{g_{\infty}}\left[-2 x+\ln \left(\frac{1+x}{1-x}\right)\right]
$$

where $x \equiv \sqrt{1-e^{-t / T}}$. It is not necessary to limit to $n=1 / 2-$ one can vary $g_{\infty}$ and $T$ independently. In other words, the above $S$ gives the scaling solution to the general equation (A5) which had Bessel functions as exact solutions. This situation is completely analogous to the case of the "harmonic oscillator" $g=g_{0}\left(1+\alpha t^{2}\right)$ : The scaling solution was valid for arbitrary $g_{0}$ and $\alpha$ for which the exact solution called for Hermite polynomials, but an exceptionally simple exact solution was obtained if $g_{0}$ and $\alpha$ satisfied a certain relationship - See Ref. [18]. In the present case the simple solution is Eq. (A8) and the required relationship is $T=1 / 4 \sqrt{g_{\infty} k A}$ in the linear regime, and $T_{L}=1 / 4 \sqrt{g_{\infty} k_{L} A_{L}}$ in the nonlinear regime.

Asymptotically, Eq. (A9) gives $s \rightarrow t \sqrt{g_{\infty}}$ which, when substituted in Eq. (22), gives $\eta \rightarrow\left(\gamma_{L} / k_{L}\right) t=\sqrt{g_{\infty} A_{L} / k_{L}} t$. The same result is obtained from the exact solution, Eq. (A8), after some algebra.

3) $g(t)=g_{n} t^{n}$. Eq. (1) reads 


$$
\frac{d^{2} \eta}{d t^{2}}-g_{n} k A t^{n} \eta=0
$$

Define $z \equiv\left(g_{n} k A\right)^{1 / 2} t^{m} / m$ where $m=1+n / 2$, let $\eta=F(t) \sqrt{t}$ and substitute in the above equation to obtain

$$
\frac{d^{2} F}{d z^{2}}+\frac{1}{Z} \frac{d F}{d z}-\left(1+\frac{1}{4 m^{2} z^{2}}\right) F=0
$$

which is the modified Bessel equation solved in terms of $I_{1 / 2 m}$ and $K_{1 / 2 m}$ [31]. For $n=1(m=3 / 2)$, which will be our $4^{\text {th }}$ and last example, the solution involves Airy functions.

It is straightforward to obtain $s_{n}(t)$ needed for the scaling solution:

$$
s_{n}(t)=\sqrt{g_{n}} \int t^{n / 2} d t=\frac{\sqrt{g_{n}}}{1+n / 2} t^{1+n / 2}
$$

which must be substituted in Eq. (22). From Eq. (25) the asymptotic bubble velocity is $\sqrt{g(t) A_{L} / k_{L}}=\sqrt{g_{n} t^{n} A_{L} / k_{L}} \sim t^{n / 2}$. The same result is obtained by analyzing the large- $Z$ behavior of $I_{1 / 2 m}$ and $K_{1 / 2 m}$.

Let us calculate the distance $\Delta x_{n}$ travelled under this acceleration:

$$
\Delta x_{n}=\int\left[\int g d t\right] d t=\frac{g_{n}}{(n+1)(n+2)} t^{n+2}
$$

so that

$$
s_{n}(t)=2 \sqrt{\Delta x_{n}} \sqrt{\frac{n+1}{n+2}} .
$$


Since $\sqrt{(n+1) /(n+2)}$ is an extremely weak function of $n$ varying between $1 / \sqrt{2} \approx 0.707$ and 1 for $0 \leq n<\infty$, we conclude that tanks undergoing different accelerations with different $n$ 's will have perturbations differing by no more than about $30 \%$ after travelling the same distance. This applies to bubbles. Of course spikes are expected to differ even less, if at all, if they scale with $\Delta x$. This mental exercise suggests an interesting and, we believe, well-defined problem: What acceleration history $g(t)$ gives minimum $\eta\left(t_{\text {end }}\right)$ if constrained to move a given $\Delta x$ by $t=t_{\text {end }}$ ? Variational calculus with Lagrange multipliers will probably give the optimum solution. Our intuition, drawing partly on Figs. 14b and 15b, favors a shock.

4) $g(t)=g_{0}+\dot{g} t$. Change variables by defining

$$
z \equiv\left(g_{0}+\dot{g} t\right) k A /(\dot{g} k A)^{2 / 3},
$$

and substitute in Eq. (1) to obtain Airy's equation

$$
\frac{d^{2} \eta}{d z^{2}}-z \eta=0
$$

whose solution is

$$
\eta(t)=\alpha A i(z)+\beta B i(z) .
$$

$A i$ and $B i$ are known as Airy functions [31,32]. The constants $\alpha$ and $\beta$ are determined by $\eta_{0}$ and $\dot{\eta}_{0}$ using the explicit forms [32]:

$$
\operatorname{Ai}(0)=\frac{B i(0)}{\sqrt{3}}=\frac{1}{3^{2 / 3} \Gamma(2 / 3)},
$$




$$
A i^{\prime}(0)=-\frac{B i^{\prime}(0)}{\sqrt{3}}=-\frac{1}{3^{1 / 3} \Gamma(1 / 3)} .
$$

Substituting these expressions into Eq. (A17) and its derivative $\dot{\eta}(t)=(\alpha / T) A i^{\prime}(z)+(\beta / T) B i^{\prime}(z)$, where $T=(\dot{g} k A)^{-1 / 3}$, we obtain

$$
\begin{aligned}
& \alpha=3^{1 / 6} \pi\left(\frac{\eta_{0}}{\Gamma(1 / 3)}-\frac{\dot{\eta}_{0} T}{3^{1 / 3} \Gamma(2 / 3)}\right), \\
& \beta=\frac{\pi}{3^{1 / 3}}\left(\frac{\eta_{0}}{\Gamma(1 / 3)}+\frac{\dot{\eta}_{0} T}{3^{1 / 3} \Gamma(2 / 3)}\right),
\end{aligned}
$$

where we have also used the relation $\Gamma(1 / 3) \Gamma(2 / 3)=2 \pi / \sqrt{3}$.

If we set $\dot{\eta}_{0}=0$ we obtain Eq. (26) which we compared with the classical solution for $g=$ const. in Sec. III.

Let us concentrate on a symmetric impulse that increases linearly from 0 to $g_{\max }=\dot{g} \tau$ by time $\tau$, then decreases linearly to 0 by time $2 \tau$. On the first leg $g=\dot{g} t, z=t(\dot{g} k A)^{1 / 3}=t / T$, and we write

$$
\begin{aligned}
& \eta(t)=\alpha_{0} A i(z)+\beta_{0} B i(z), \\
& \dot{\eta}(t)=\frac{\alpha_{0}}{T} A i^{\prime}(z)+\frac{\beta_{0}}{T} B i^{\prime}(z),
\end{aligned}
$$

with

$$
\alpha_{0}=3^{1 / 6} \pi \eta_{0} / \Gamma(1 / 3)
$$




$$
\beta_{0}=\pi \eta_{0} / 3^{1 / 3} \Gamma(1 / 3),
$$

which follow from Eq. (A19a,b) after setting $\dot{\eta}_{0}=0$.

On the second leg between $\tau$ and $2 \tau, g=\dot{g}(2 \tau-t)$, and

$$
\begin{aligned}
& \eta(t)=\alpha_{2} A i\left(z_{2}\right)+\beta_{2} \operatorname{Bi}\left(z_{2}\right), \\
& \dot{\eta}(t)=-\frac{\alpha_{2}}{T} A i^{\prime}\left(z_{2}\right)-\frac{\beta_{2}}{T} B i^{\prime}\left(z_{2}\right),
\end{aligned}
$$

where $z_{2} \equiv(2 \tau-t) / T$. The constants $\alpha_{2}$ and $\beta_{2}$ are determined by the initial $(t=\tau)$ conditions of the second leg, $\eta(\tau)$ and $\dot{\eta}(\tau)$, which are the final $(t=\tau)$ conditions on the first leg and are therefore given by Eq. (A20a,b):

$$
\begin{aligned}
\eta(\tau) & =\alpha_{0} A i(\tau / T)+\beta_{0} \operatorname{Bi}(\tau / T) \\
& =\alpha_{2} \operatorname{Ai}(\tau / T)+\beta_{2} \operatorname{Bi}(\tau / T), \\
\dot{\eta}(\tau) & =\frac{\alpha_{0}}{T} A i^{\prime}(\tau / T)+\frac{\beta_{0}}{T} B i^{\prime}(\tau / T) \\
& =-\frac{\alpha_{2}}{T} A i^{\prime}(\tau / T)-\frac{\beta_{2}}{T} B i^{\prime}(\tau / T) .
\end{aligned}
$$

From these two equations we obtain

$$
\alpha_{2}=\alpha_{0}+2 \pi \operatorname{Bi}(\tau / T)\left[\alpha_{0} A i^{\prime}(\tau / T)+\beta_{0} B i^{\prime}(\tau / T)\right] \text {, }
$$

and

$$
\beta_{2}=\beta_{0}-2 \pi A i(\tau / T)\left[\alpha_{0} A i^{\prime}(\tau / T)+\beta_{0} B i^{\prime}(\tau / T)\right] .
$$


We used the Wronskian, $A i B i^{\prime}-B i A i^{\prime}=1 / \pi$, valid for any $z$, to simplify the result.

Since $\alpha_{2}$ and $\beta_{2}$ are now known, the evolution along the second leg given by Eq. (A22) is also known for any time $\tau \leq t \leq 2 \tau$. In particular, at the end of the pulse, i.e., at $t=2 \tau$ or $z_{2}=0$ :

$$
\begin{aligned}
& \eta(2 \tau)=\alpha_{2} A i(0)+\beta_{2} B i(0), \\
& \dot{\eta}(2 \tau)=-\frac{\alpha_{2}}{T} A i^{\prime}(0)-\frac{\beta_{2}}{T} B i^{\prime}(0) .
\end{aligned}
$$

These values of $\eta$ and $\dot{\eta}$ can be used as "initial” conditions for the third and last leg of the acceleration where we maintain $g=0$ (see Fig. 1c) and therefore the solution is given by Eq. (15) for $t \geq 2 \tau$.

The main results of this example are the above two equations giving the amplitude and its growth rate at the end of the pulse. After some algebra using Eqs. (A18), (A21), and (A24), a relatively simple form emerges:

$$
\eta(2 \tau)=\eta_{0}\left\{1+\frac{\pi}{\sqrt{3}}\left[B i-\sqrt{3} A i\left[B i^{\prime}+\sqrt{3} A i^{\prime}\right]\right\},\right.
$$

and

$$
\dot{\eta}(2 \tau)=\frac{2 \eta_{0}}{T}\left(\frac{\pi}{3^{1 / 3} \Gamma(1 / 3)}\right)^{2}[B i+\sqrt{3} A i]\left[B i^{\prime}+\sqrt{3} A i^{\prime}\right]
$$

with all Airy functions and their derivatives evaluated at $\tau / T$. The reader will recognize some of the bracketed terms above as $\eta(\tau)$ and $\dot{\eta}(\tau)$. In particular, 


$$
\dot{\eta}(2 \tau) \propto \eta(\tau) \dot{\eta}(\tau)
$$

The above expressions are valid for arbitrary $\tau / T$, the normalized half-width of the pulse. To obtain the small- $\tau / T$ relations given in [18], expand Eqs. (A26a,b) for $\tau / T<<1$. This is a somewhat laborious procedure because most of the leading or next to the leading terms vanish (e.g., $B i^{\prime}(0)+\sqrt{3} A i^{\prime}(0)=0$ ) and one must go to higher order Taylor expansions to obtain a non-vanishing contribution. A third-order expansion is needed for $\eta(2 \tau)$ and a sixth-order expansion for $\dot{\eta}(2 \tau)$. The final results, however, are exceptionally simple:

$$
\eta(2 \tau)=\eta_{0}\left(1+(\tau / T)^{3}+\ldots\right)
$$

and

$$
\dot{\eta}(2 \tau)=\eta_{0}\left(\tau^{2} / T^{3}\right)\left(1+7(\tau / T)^{3} / 30+\ldots\right) .
$$

Using the definition $T=(\dot{g} k A)^{-1 / 3}$ and the relation

$$
\Delta \mathrm{v}=\int_{0}^{2 \tau} g d t=g_{\max } \tau=\dot{g} \tau^{2}
$$

we write

$$
\begin{aligned}
& \eta(2 \tau)=\eta_{0}(1+\Delta \mathrm{v} k A \tau+\ldots), \\
& \dot{\eta}(2 \tau)=\eta_{0} \Delta \mathrm{v} k A(1+7 \Delta \mathrm{v} k A \tau / 30+\ldots),
\end{aligned}
$$

as reported in [18]. 
We now turn to the scaling solution. On the first leg where $g=\dot{g} t$ Eq. (A12)

with $n=1$ gives

$$
s(t \leq \tau)=\frac{2 \sqrt{\dot{g}}}{3} t^{3 / 2}
$$

On the second leg where $g=\dot{g}(2 \tau-t)$,

$$
s(t \geq \tau)=\frac{4}{3} \sqrt{\dot{g}} \tau^{3 / 2}\left[1-\frac{1}{2}(2-t / \tau)^{3 / 2}\right] .
$$

In particular,

$$
s(2 \tau)=\frac{4}{3} \sqrt{\dot{g}} \tau^{3 / 2}
$$

By symmetry, $s(2 \tau)=2 s(\tau)$.

On either leg the linear- $\eta$ scaling solution is

$$
\eta(t)=\eta_{0} \cosh (s \sqrt{k A})
$$

Therefore,

$$
\eta(2 \tau)=\eta_{0} \cosh \left(\frac{4}{3} \sqrt{\dot{g} k A \tau^{3}}\right)=\eta_{0} \cosh \left(\frac{4}{3}(\tau / T)^{3 / 2}\right)
$$

When $\tau / T<<1$, this scaling solution is

$$
\eta(2 \tau)=\eta_{0}\left(1+\frac{8}{9}(\tau / T)^{3}+\ldots\right)=\eta_{0}\left(1+\frac{8}{9} \Delta v k A \tau+\ldots\right)
$$

to be compared with the exact expansion, Eq. (A30a), giving 1 instead of 8/9 as the coefficient of the second term. This is surprisingly good agreement given the lengthy 
derivation of the exact expansion and given that $g=0$ at $t=2 \tau$ where one cannot justify the scaling solution. This is the reason why one cannot apply the scaling solution to $\dot{\eta}$ because it gives $\dot{\eta} \sim \sqrt{g(t)}=0$ at $t=2 \tau$, in contrast to the exact result Eq. (A26b) or its expansion Eq. (A30b).

Of course the expansions get poorer as the pulse gets wider. In Table I we compare equations (A26a), (A28a), (A34), and (A35).

We believe the small- $\tau$ expansions, Eqs. (A28) or (A35), have a wider application and are not limited to linearly increasing/decreasing impulses only. The reason is the following: We considered an impulse that increases exponentially with time, $g \sim e^{t / T}-1$ until $t=\tau$, and then decreases exponentially to 0 at $t=2 \tau$. The exact solution involves Bessel functions (see example 2). The scaling solution, however, is quite simple and involves an elementary integration only. We omit the details. The point we wish to make is that when we expand $\eta(2 \tau)$ for small $\tau / T$ and of course define $\Delta \mathrm{v}=\int_{0}^{2 \tau} g d t$, etc, corresponding to this exponential impulse, we obtain the same equation as (A35), with the 8/9 factor. Apparently, when the impulse is very narrow, it does not matter how $g_{\max }$ is reached, linearly or exponentially or otherwise.

Asymptotic nonlinear solutions are obtained by the now familiar procedure of $k \rightarrow k_{L}, A \rightarrow A_{L}$, and taking the logarithm. The case $g=\dot{g} t$ was discussed in Ref. [18] and will not be repeated here. Both the exact and the scaling solutions give $\left(\dot{g} A_{L} / k_{L}\right)^{1 / 2} t^{1 / 2}=\sqrt{g(t) A_{L} / k_{L}}$ as the asymptotic bubble velocity $\dot{\eta}_{\infty}$. 
We do not investigate other examples. The "harmonic oscillator" was discussed in [18]. Other well known QM potentials $V(x)$ such as the Coulomb, Wood-Saxon, Hulthén, Pöschl-Teller, etc, can be converted to acceleration histories $g(t)$ and solved by similar techniques. We concentrated heavily on the linear potential because it is used in so many experiments and is probably the next logical step after the classic RT and RM instabilities.

\section{Figure Captions}

Fig. 1. Three acceleration profiles similar to the ones used in LEM experiments $[5,6]$ and used in CALE simulations in this work.

Fig. 2. The bubble amplitude $\eta(t)$, starting from $\eta_{0}=\eta^{*} / 2=0.065 \mathrm{~cm}$, for the three acceleration profiles shown in Fig. 1. The dashed lines correspond to CALE simulations which we label level 1 . The two continuous lines, undistinguishable in these figures, correspond to level 2 and level 3 solutions. The level 2 solution is obtained from the coupled equations (5) and (6). The level 3 solution is obtained from Eq. (1) for $\eta \leq \eta^{*}$, after which we use Eq. (10). Analytic level 3 solutions in terms of Airy functions and level 4 scaling solutions in terms of elementary functions can also be written down (see the Appendix).

Fig. 3. Quasi-constant acceleration (in arbitrary units) given by Eq. (29), and the corresponding amplitudes as calculated by CALE for $g_{\infty}=35 g_{E}, T=1.2 \mathrm{~ms}$, and the analytic solution Eq. (30), starting with $\eta_{0}=\eta^{*}=0.13 \mathrm{~cm}$. 
Fig. 4. Same as Fig. 3 with $\eta_{0}=4 \eta^{*}=0.52 \mathrm{~cm}$. The level 2 model, based on Eqs. (5) and (6), fails for amplitudes $\eta_{0}>\left(\eta_{0}\right)_{\max }$ where, from Eq. (9), $\left(\eta_{0}\right)_{\max } \approx 0.48 \mathrm{~cm}$. The level 3 model, using Eq. (10) for all $\eta_{0} \geq \eta^{*}$, gives a reasonable result. Fig. 5. The idealized acceleration given by Eq. (29) (thick line) and the code-calculated interface acceleration (thin dashed line) showing oscillations around an average value. Imposing deliberately large oscillations as in Eq. (31) with $\varepsilon=1$ and $\omega=2 \pi / 10 \mathrm{~ms}^{-1}$ varies $g(t)$ by $\pm 100 \%$ around its average value of $35 g_{E}$.

Fig. 6. The deviation in bubble amplitude with and without the large oscillations shown in Fig. 5 with $\varepsilon=1$. Eq. (32) defines $\delta(t ; \varepsilon)$.

Fig. 7. Acceleration profile for a possible double-impulse experiment. The impulses are equal and opposite, bringing the tank to rest at $30 \mathrm{~ms}$.

Fig. 8. The "bubble" amplitude for the double-impulse experiment with $g(t)$ shown in Fig. 7 (repeated here in dashed line and arbitrary units). The initial amplitude is 0.065 $\mathrm{cm}$. The bubble turns into a spike after $30 \mathrm{~ms}$, the point where Eqs. (5) and (10) both fail. Snapshots in Fig. 9.

Fig. 9. Snapshots of the interface as calculated by CALE for the double-impulse acceleration history shown in Fig. 7, starting with $\eta_{0}=0.13 \mathrm{~cm}$. We have included the distance travelled in the first 3 snapshots. At $30 \mathrm{~ms}$ the tank comes to rest $6.2 \mathrm{~cm}$ below its initial position while the perturbation continues to evolve.

Fig. 10. An acceleration history with a weaker reshock that can induce freeze-out, i.e., make $\eta(t)=$ const. See Fig. 11 . 
Fig. 11. The bubble amplitude calculated by CALE, by Eqs. (5)+(6), and by Eq. (10), using the acceleration profile $g(t)$ shown in Fig. 10. CALE predicts complete freeze-out (we iterated on $g(t)$ to obtain this result). Eqs. (5)+(6) or Eq. (10) predict slow increase or decrease. The calculations start with $\eta_{0}=0.065 \mathrm{~cm}$. Without reshock $\eta$ would continue to grow, $\eta \sim \ln t$, as in Eq. (15).

Fig. 12. Bubble amplitudes as functions of $s$ defined by Eq. (19). The 4 cases A, B, C, and D correspond to Figs. 1a, 1b, 1c, and Eq. (29), respectively (see also Fig. 3 for the quasi-constant acceleration D). Initial amplitude $\eta_{0}=0.13 \mathrm{~cm}$. Curve E is Eq. (22). Bubbles appear to scale with $S$ except after $g=0$ (curve C after $s \approx 5 \mathrm{~cm}^{1 / 2}$ ).

Fig. 13. Three acceleration profiles used for testing the scaling hypothesis with CALE simulations. A is a constant acceleration at $70 g_{E}$. B has $g=g_{3} t^{3}$ with $g_{3}=0.06 g_{E} / \mathrm{ms}^{3}$. C is an impulsive acceleration reaching $500 g_{E}$ in $1 \mathrm{~ms}$ and returning to zero at $t=2 \tau=2 \mathrm{~ms}$. Accelerations in units of $g_{E}$, displacements in $\mathrm{cm}$, and scaling variables in $\mathrm{cm}^{1 / 2}$ are plotted in diagrams a, b, and c respectively, all as functions of time in milliseconds.

Fig. 14. Bubble $(\eta>0)$ and spike $(\eta<0)$ evolutions as calculated by CALE for the three acceleration profiles A, B, and C displayed in Fig. 13. They are plotted as functions of time in (a), $\Delta x$ in (b), and $s$ in (c). Bubbles appear to scale with $s$, while spikes prefer $\Delta x$. The impulsive acceleration $\mathrm{C}$ does not scale after $g=0$.

Fig. 15. Same as Fig. 14 with the hexane $\left(\rho=0.66 \mathrm{~g} / \mathrm{cm}^{3}\right)$ in the tank replaced by air ( $\left.\rho=0.0012 \mathrm{~g} / \mathrm{cm}^{3}\right)$, hence $A \approx 1$. 
Fig. 16. Bubble and spike amplitudes calculated by CALE (thin dashed line) and by Eq. (36) (thick continuous lines). The acceleration is the negative of the impulse C in Fig. 13, and $A \approx 1$. The insets show the heavy fluid at $t=0$ and $t=24 \mathrm{~ms}$.

Fig. 17. Same as Fig. 16 with an equal and opposite reshock added at $10 \mathrm{~ms}$, as shown by the thin dashed line in arbitrary units. CALE simulations starting with $\eta_{0}=0.13 \mathrm{~cm}$. The spike reaches a magnitude of $9.5 \mathrm{~cm}$ by $24 \mathrm{~ms}$, compared with $4.3 \mathrm{~cm}$ for the shockonly case.

Fig. 18. Comparison of two tanks with a single shock only (left figure, $\eta$ 's in Fig. 16) and for shock+reshock (right figure, $\eta$ 's in Fig. 17), both at 24 ms. The position ( 15 $\mathrm{cm})$ and speed $(\sim 0.68 \mathrm{~cm} / \mathrm{ms})$ of the tip of the jet in the laboratory are approximately the same in both cases. The tank at left is moving up at $0.49 \mathrm{~cm} / \mathrm{ms}$, hence $\dot{\eta}^{\text {s }}=0.49-0.68=-0.19 \mathrm{~cm} / \mathrm{ms}$. The tank at right is at rest, hence $\dot{\eta}^{s}=0-0.68=-0.68 \mathrm{~cm} / \mathrm{ms}$. Both tanks started with $\eta_{0}=0.13 \mathrm{~cm}, y_{0}=0$ defining the laboratory position of the initial interface, carrying a reservoir of heavy fluid $4.4 \mathrm{~cm}$ thick.

\section{$\underline{\text { Table Caption }}$}

Table I. The ratio $\eta(2 \tau) / \eta_{0}$, often called the growth factor, for an acceleration that increases linearly with time reaching a maximum $g_{\max }$ at $t=\tau$ and returning to zero at $t=2 \tau$. Exact results are obtained from Eq. (A26a), whose expansion for small $\tau / T$ is Eq. (A28a). The scaling solution is Eq. (A34) and its expansion is Eq. (A35). 


\section{REFERENCES}

[1] Lord Rayleigh, Scientific Papers, 2, ( Dover, New York, 1965); G. I. Taylor, Proc. R. Soc. London Ser. A 201, 192 (1950).

[2] R. D. Richtmyer, Commun. Pure Appl. Math. 13, 297 (1960); E. E. Meshkov, Fluid Dyn. 4, 101 (1969).

[3] J. D. Lindl, Inertial Confinement Fusion (Springer, New York, 1998).

[4] K. I. Read, Physica 12D, 45 (1984).

[5] G. Dimonte and M. Schneider, Phys. Rev. E 54, 3740 (1996).

[6] G. Dimonte and M. Schneider, Phys. Fluids 12, 304 (2000).

[7] J. W. Jacobs and J. M. Sheeley, Phys. Fluids 8, 405 (1996).

[8] C. E. Niederhaus and J. W. Jacobs, J. Fluid Mech. 485, 243 (2003).

[9] P. R. Chapman and J. W. Jacobs, Phys. Fluids 18, 074101 (2006).

[10] K. O. Mikaelian, Phys. Rev. E 78, 015303 (2008).

[11] R. E. Tipton, CALE Users Manual (unpublished).

[12] D. Layzer, Astrophys. J. 122, 1 (1955).

[13] K. O. Mikaelian, Phys. Rev. Lett. 80, 508 (1998).

[14] Q. Zhang, Phys. Rev. Lett. 81, 3391 (1998).

[15] V. N. Goncharov, Phys. Rev. Lett. 88, 134502 (2002).

[16] N. A. Inogamov and A. M. Oparin, JETP 97, 1168 (2003). 
[17] K. O. Mikaelian, Phys. Fluids 21, 024103 (2009).

[18] K. O. Mikaelian, Phys. Rev. E 79, 065303 (2009).

[19] O. Sadot et al., Phys. Rev. Letters 80, 1654 (1998).

[20] Q. Zhang and S. Sohn, Z. Angew. Math. Phys. 50, 1 (1999).

[21] M. Vetter and B. Sturtevant, Shock Waves 4, 247 (1995).

[22] F. Poggi, M. H. Thorembey, and G. Rodriguez, Phys. Fluids 10, 2698 (1998).

[23] B. J. Balakumar et al., Phys. Fluids 20, 124103 (2008).

[24] K. O. Mikaelian, Phys. Rev. A 31, 410 (1985).

[25] A. A. Charakhch’yan, Plasma Phys. Controlled Fusion 43, 1169 (2001).

[26] E. Leinov et al., J. Fluid Mech. 626, 449 (2009).

[27] W. P. Walters and J. A. Zukas, Fundamentals of Shaped Charges (CMC, Maryland, 1998).

[28] M. B. Zellner and W. T. Buttler, App. Phys. Letters 93, 114102 (2008).

[29] G. Birkhoff and E. H. Zarantonello, Jets, Wakes, and Cavities (Academic Press, New York, 1957).

[30] S. I. Abarzhi, K. Nishihara, and R. Rosner, Phys. Rev. E 73, 036310 (2006).

[31] E. T. Whittaker and G. N. Watson, A Course of Modern Analysis (Cambridge University Press, Cambridge, 1963).

[32] A. Erdélyi, Asymptotic Expansions, (Dover, New York, 1956). 


\section{TABLE I}

\begin{tabular}{|c|c|c|c|c|}
\hline \multicolumn{1}{|c|}{$\tau / T$} & Eq. (A26a) & Eq. (A28a) & Eq. (A34) & Eq. (A35) \\
\hline \hline 0 & 1 & 1 & 1 & 1 \\
\hline \hline 0.5 & 1.127 & 1.125 & 1.113 & 1.111 \\
\hline 1.0 & 2.159 & 2.000 & 2.029 & 1.889 \\
\hline 1.5 & 6.466 & 4.375 & 5.834 & 4.000 \\
\hline \hline 2.0 & 24.541 & 9.000 & 21.728 & 8.111 \\
\hline
\end{tabular}




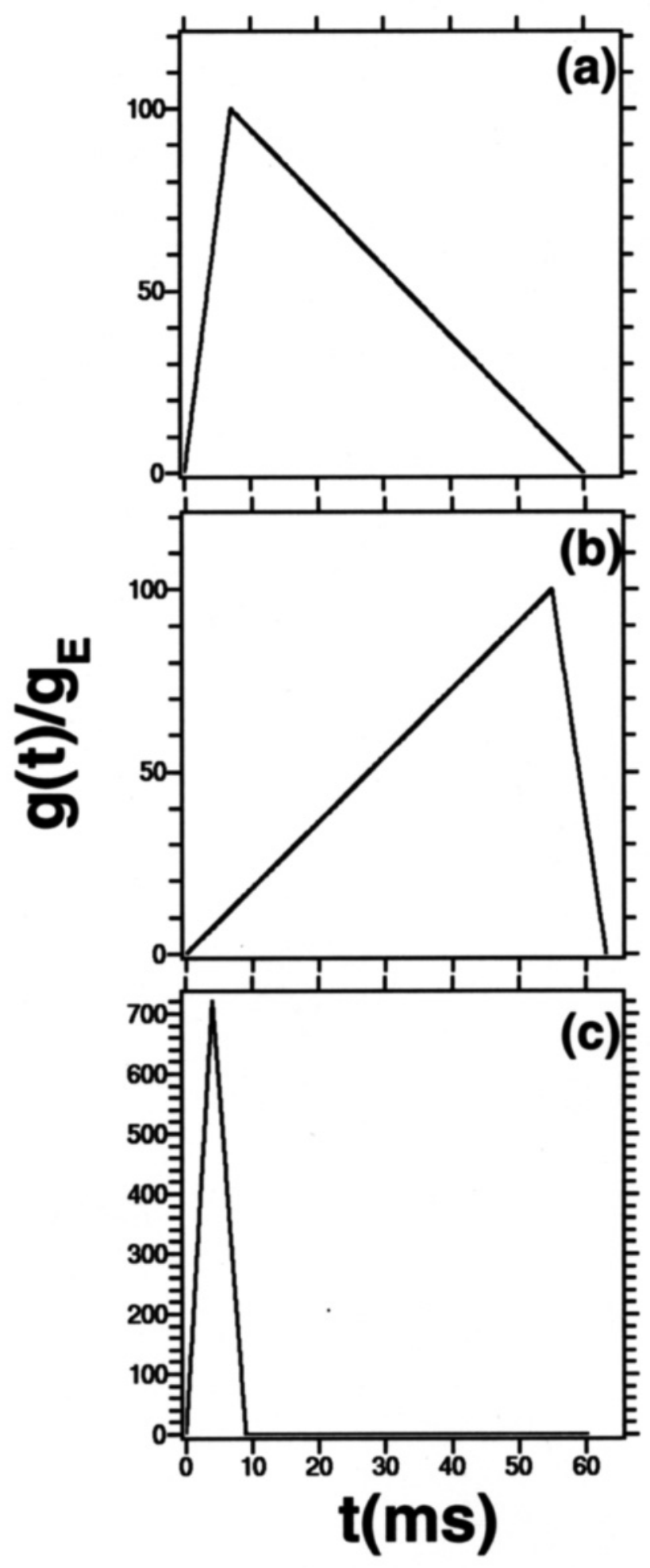

Fig. 1 


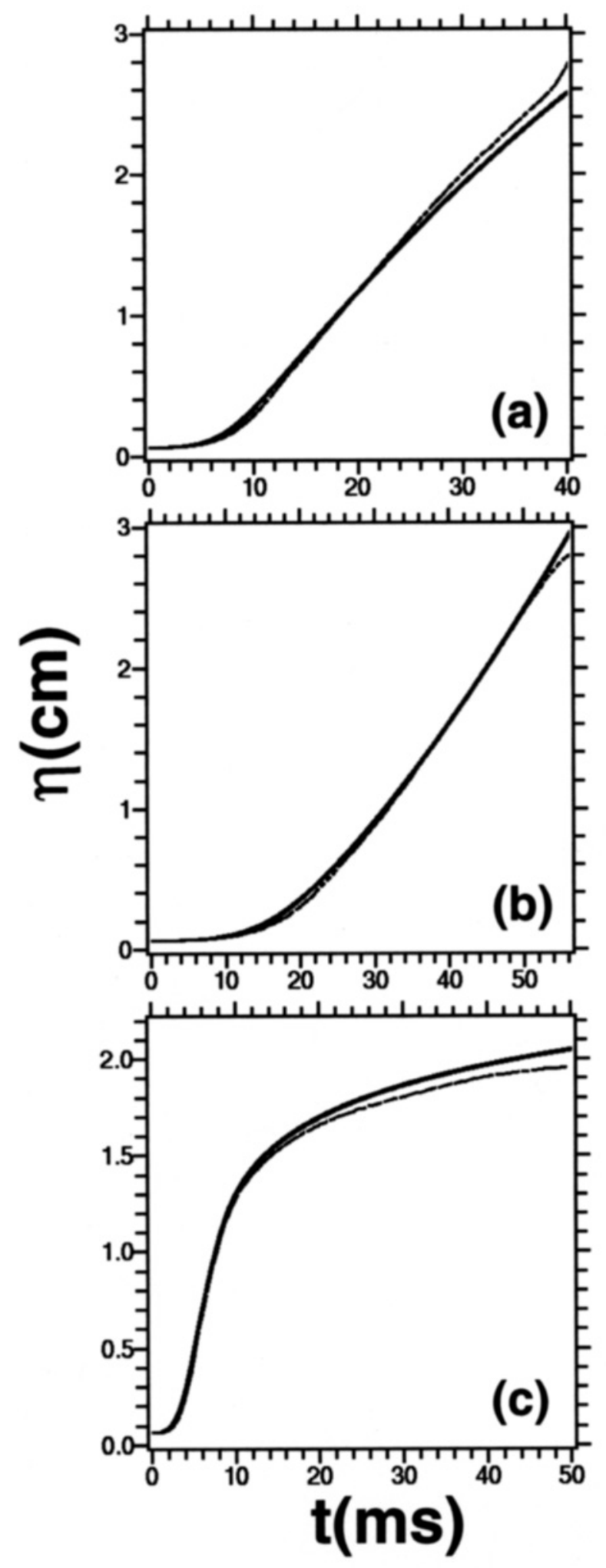

Fig. 2 


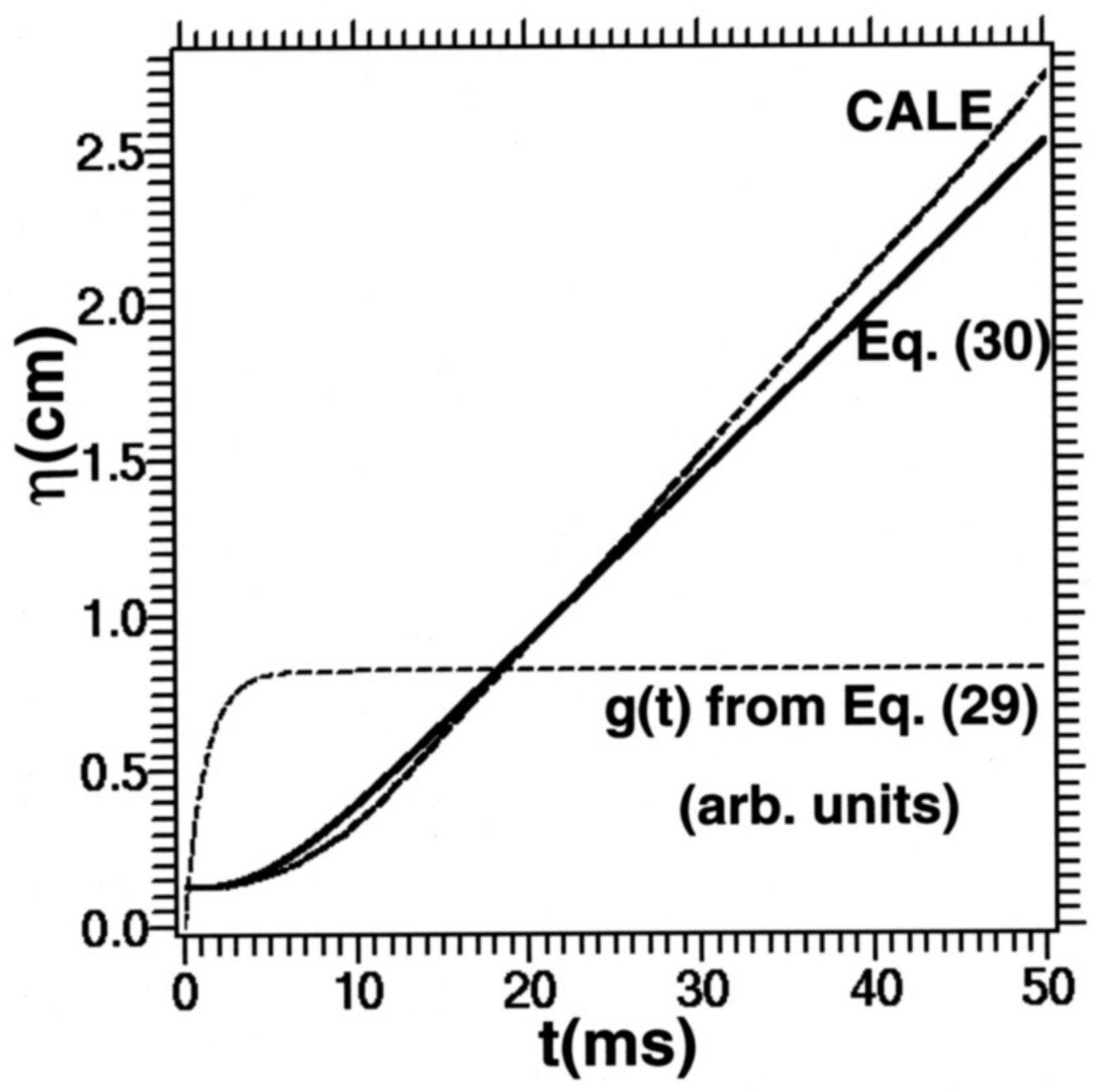

Fig. 3 


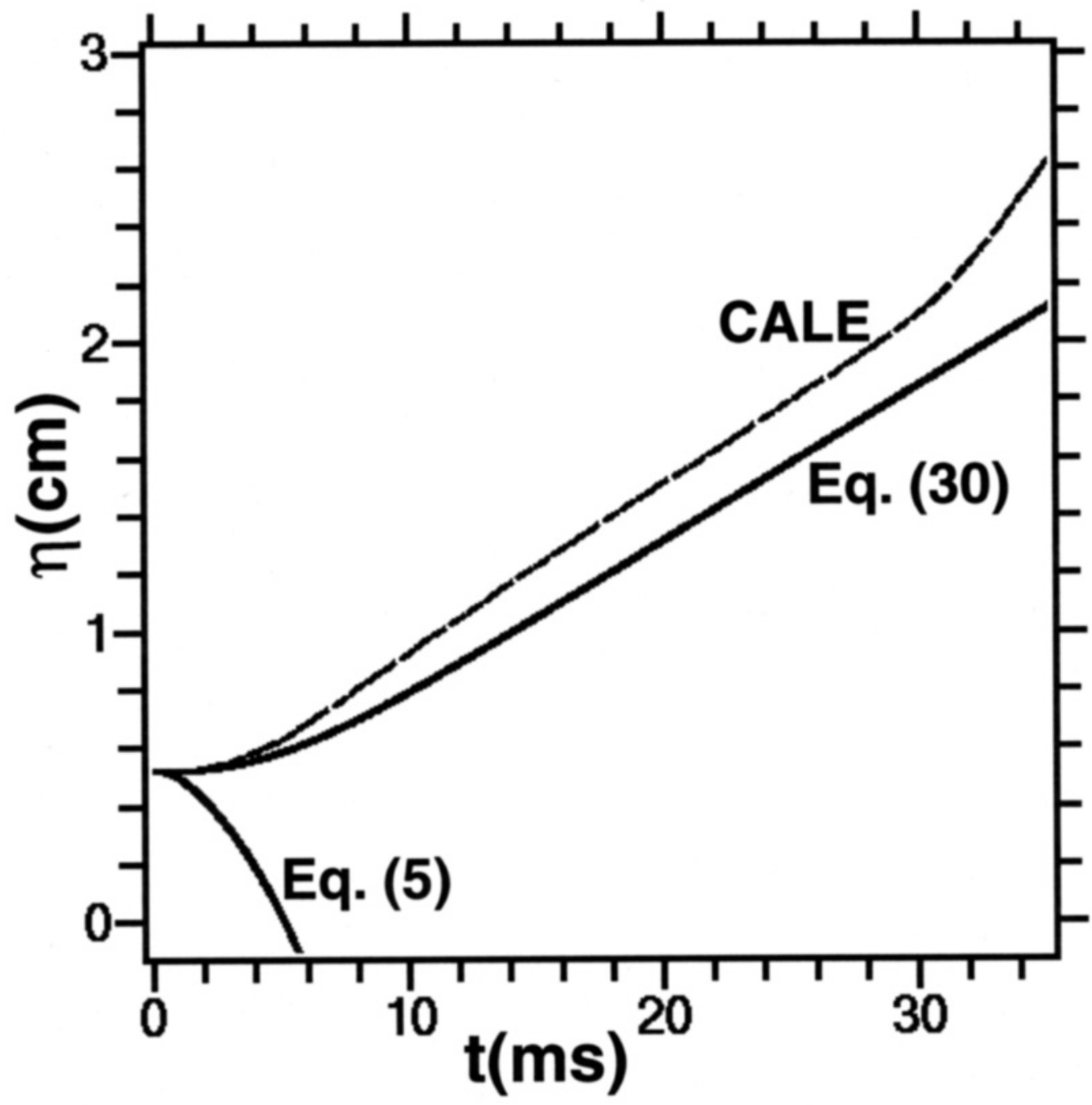

Fig. 4 


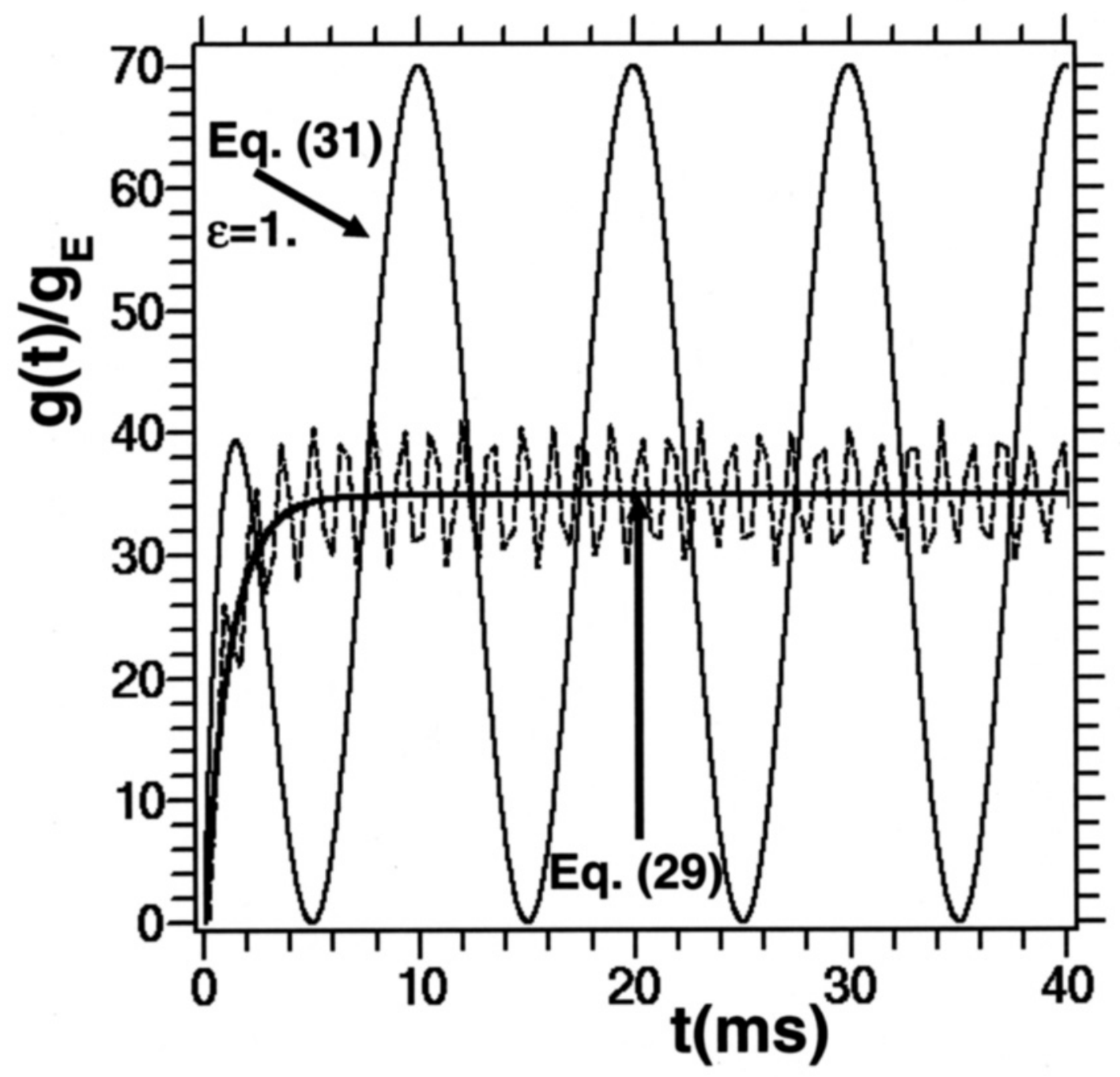

Fig. 5 


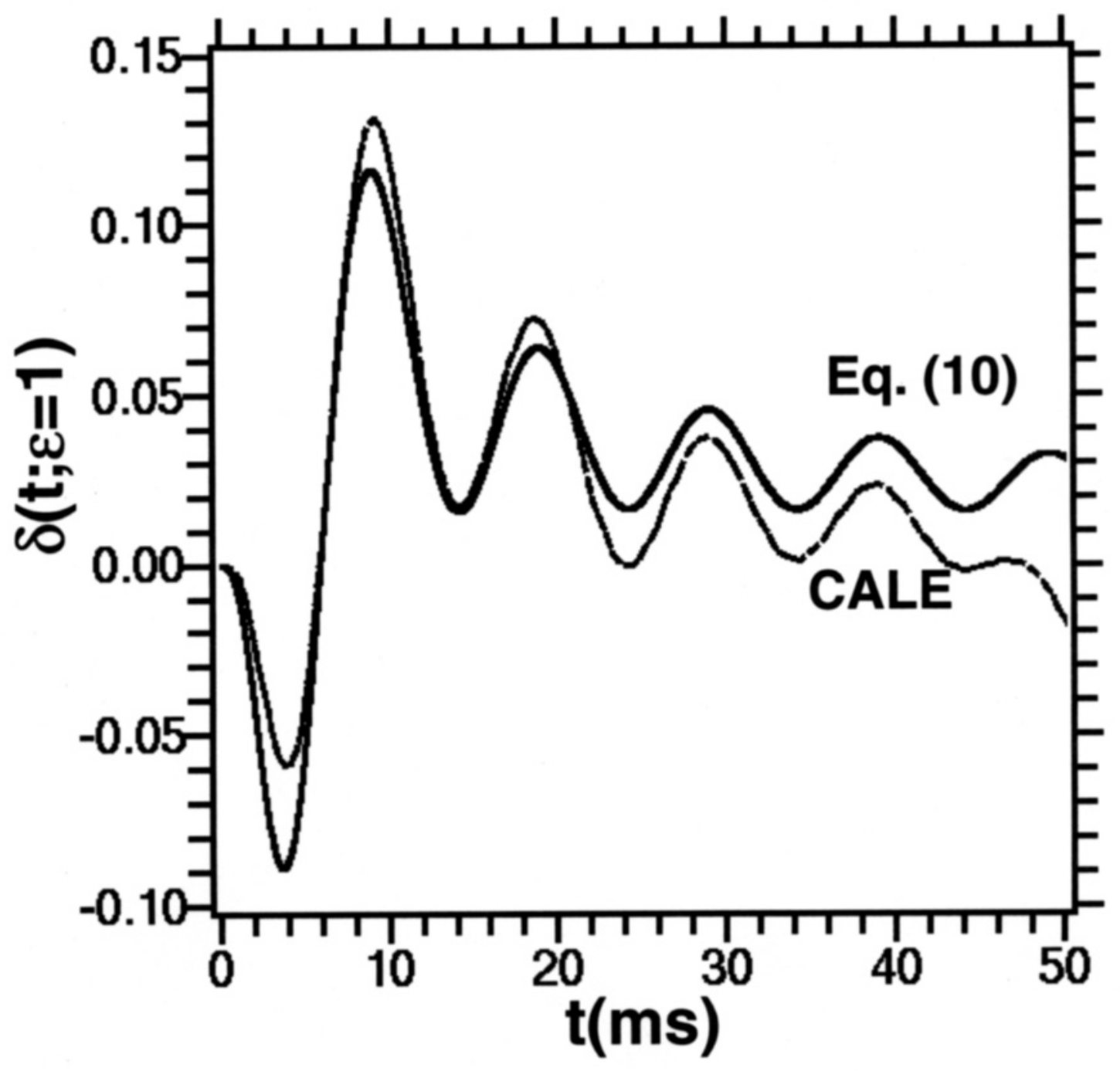

Fig. 6 


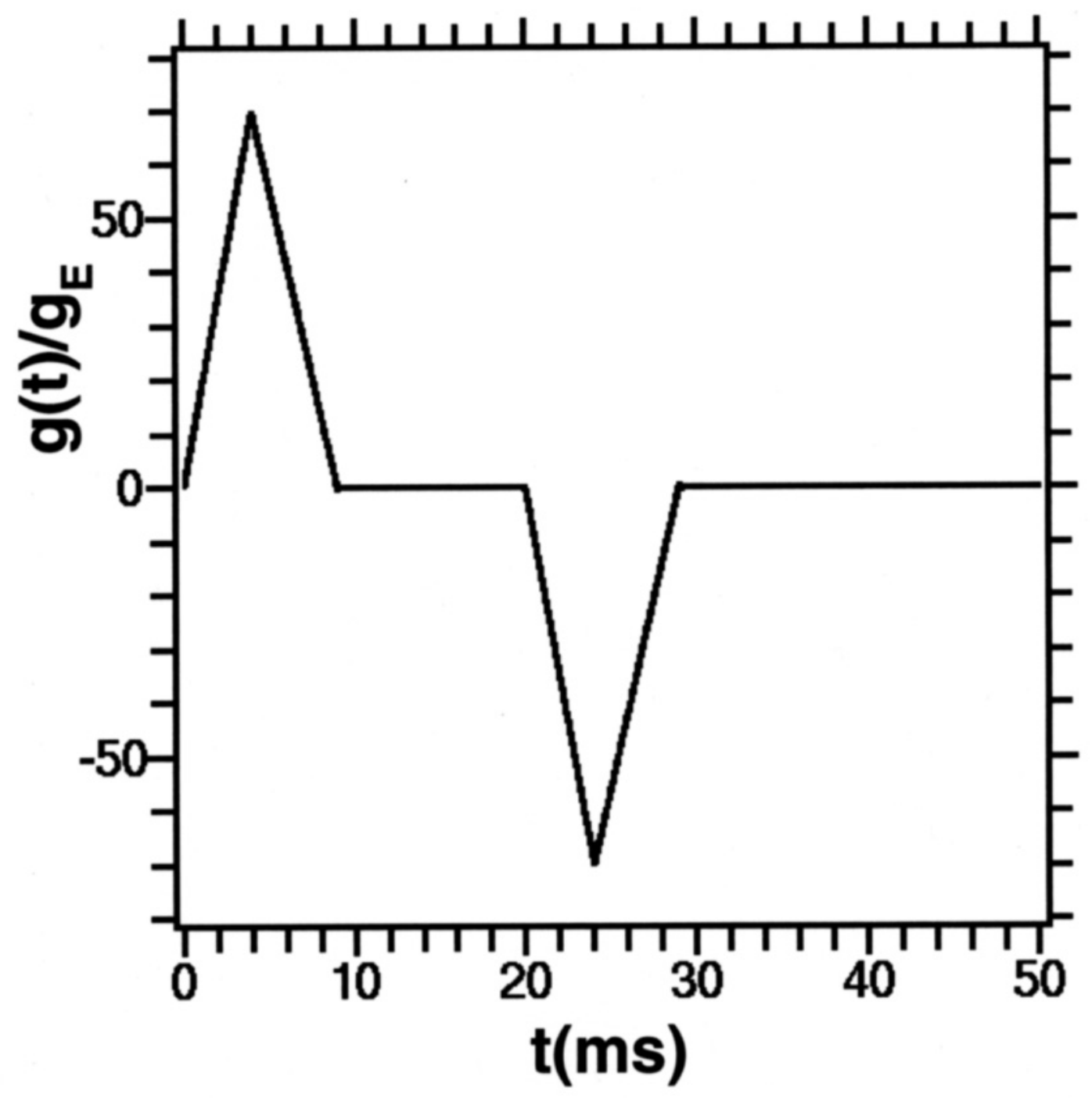

Fig. 7 


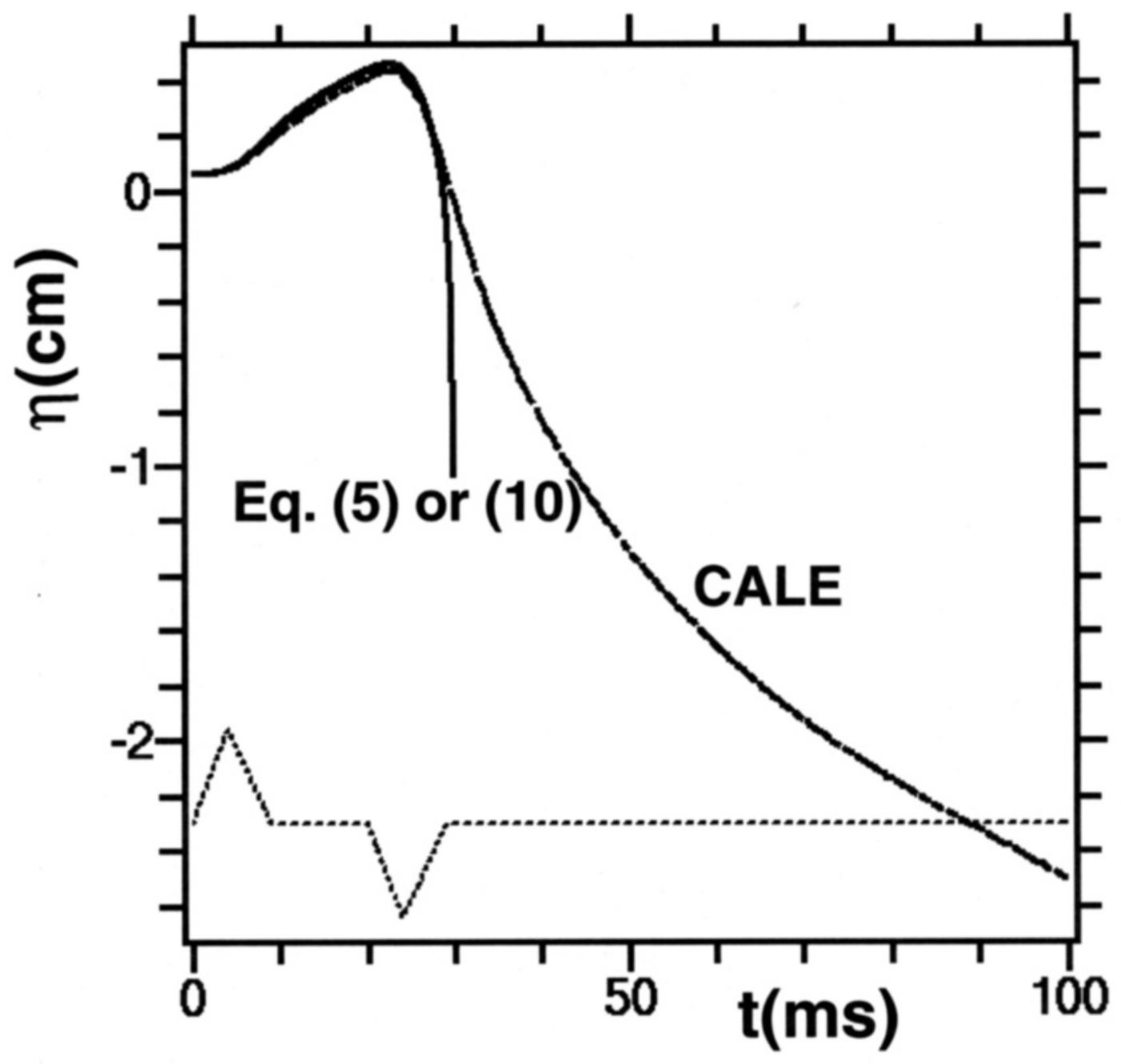

Fig. 8 

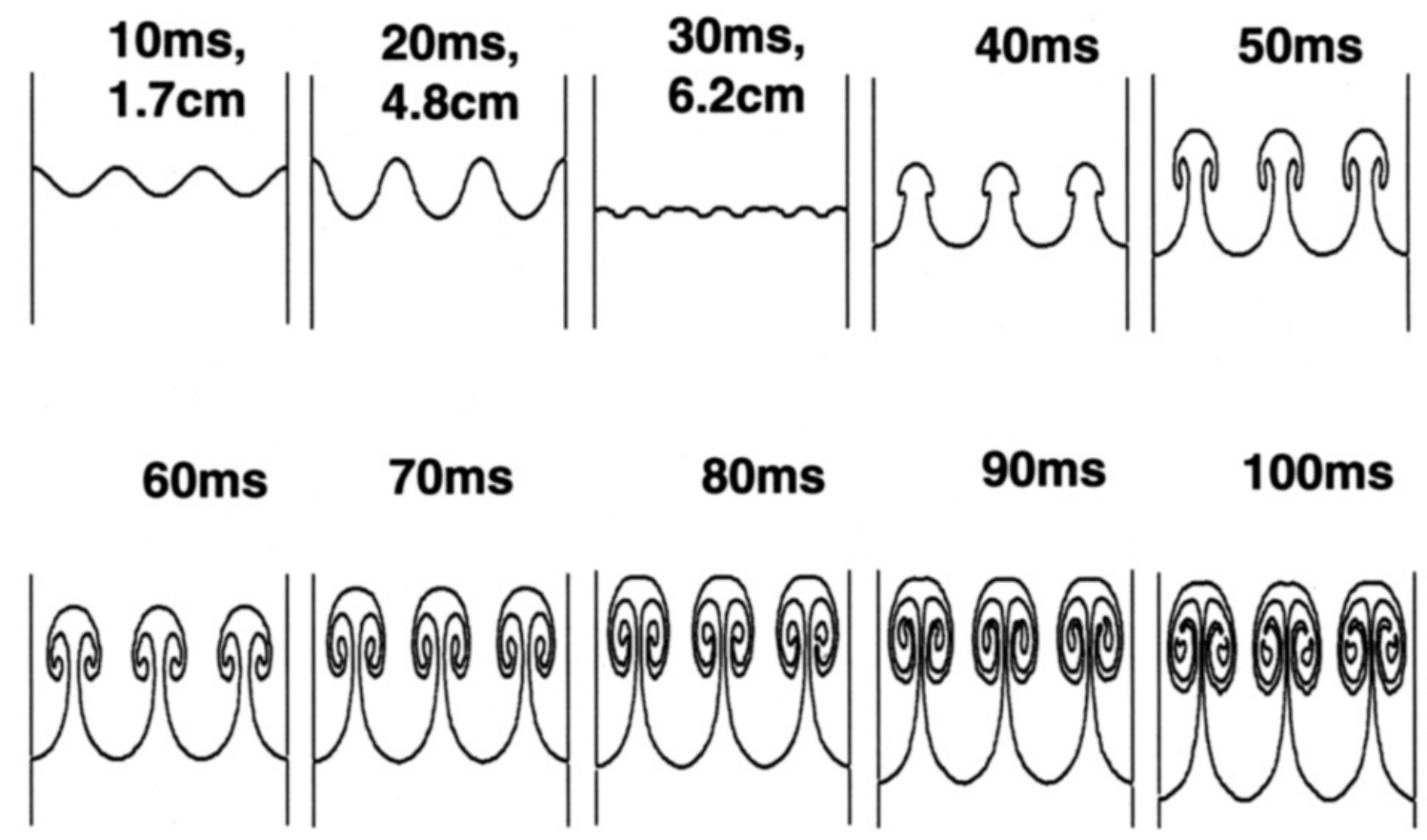

Fig. 9 


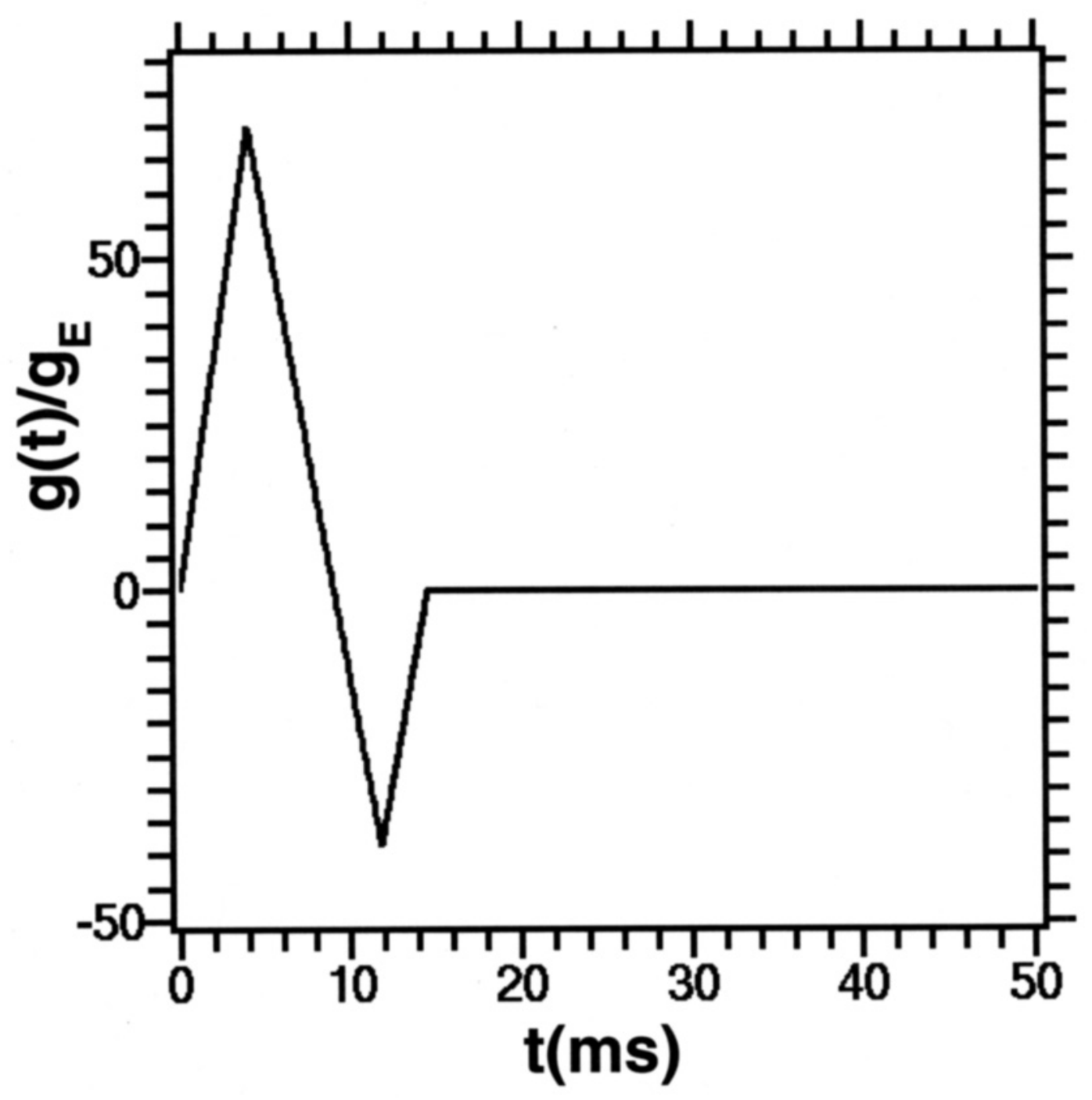

Fig. 10 


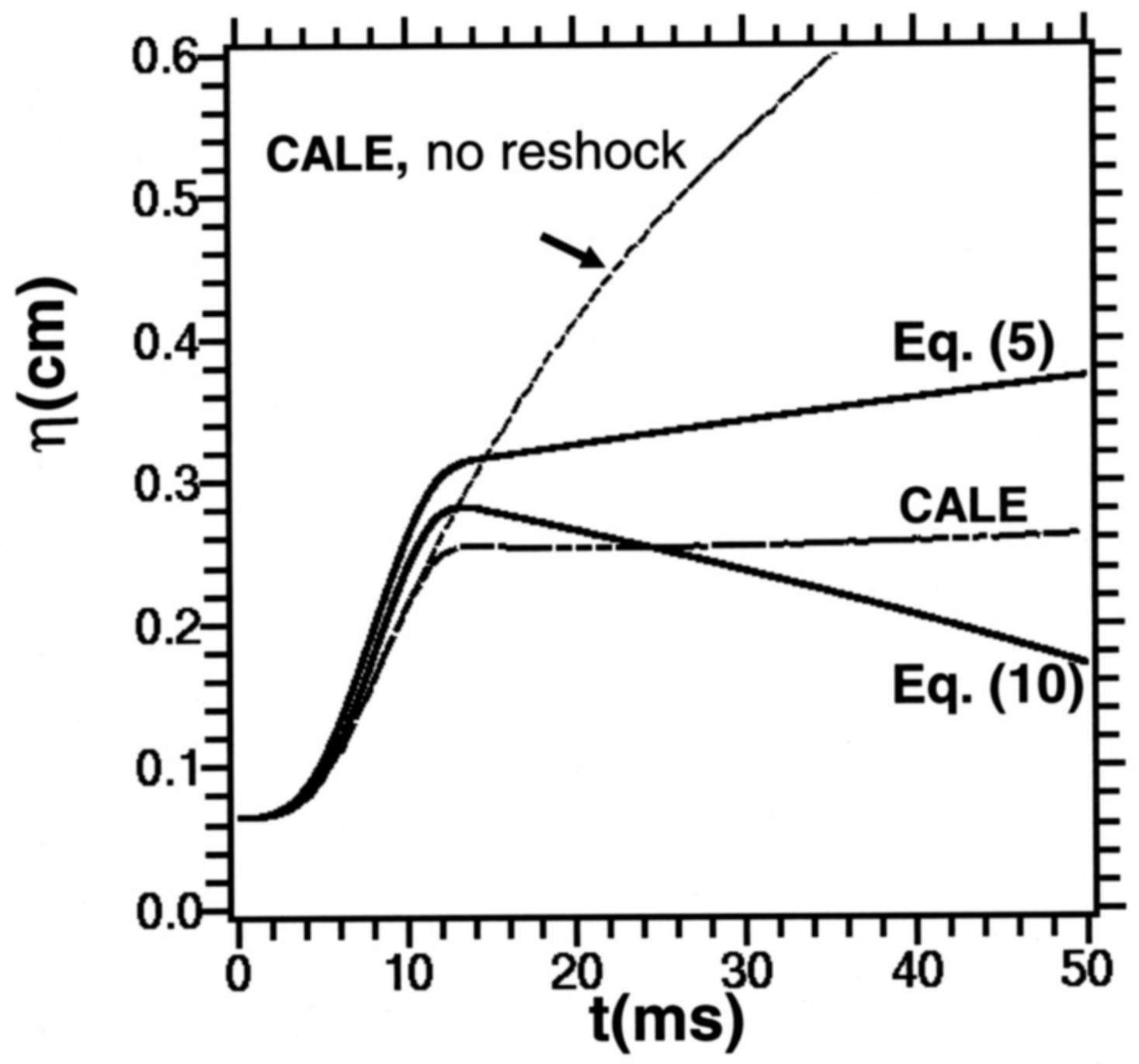

Fig. 11 


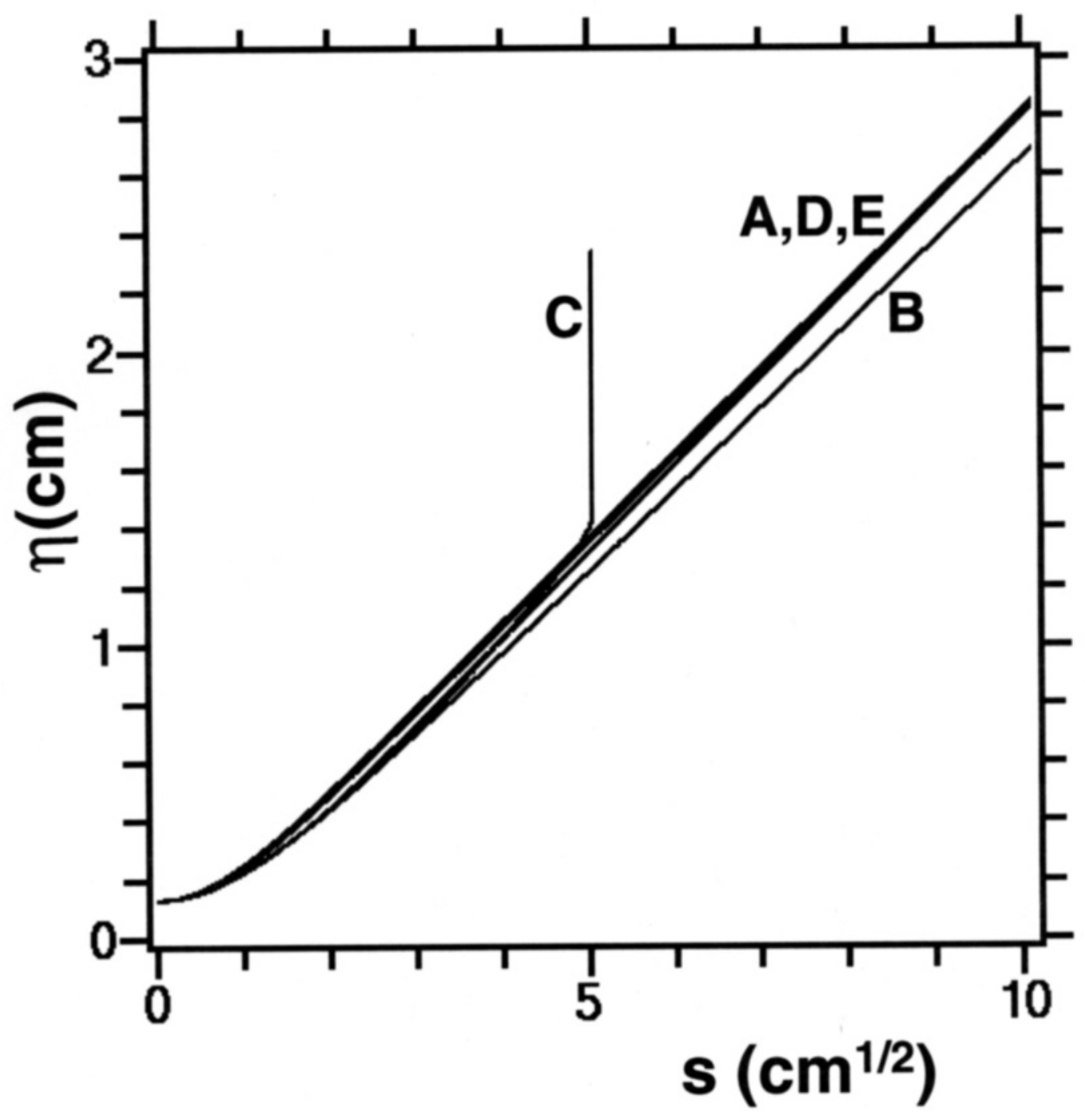

Fig. 12 

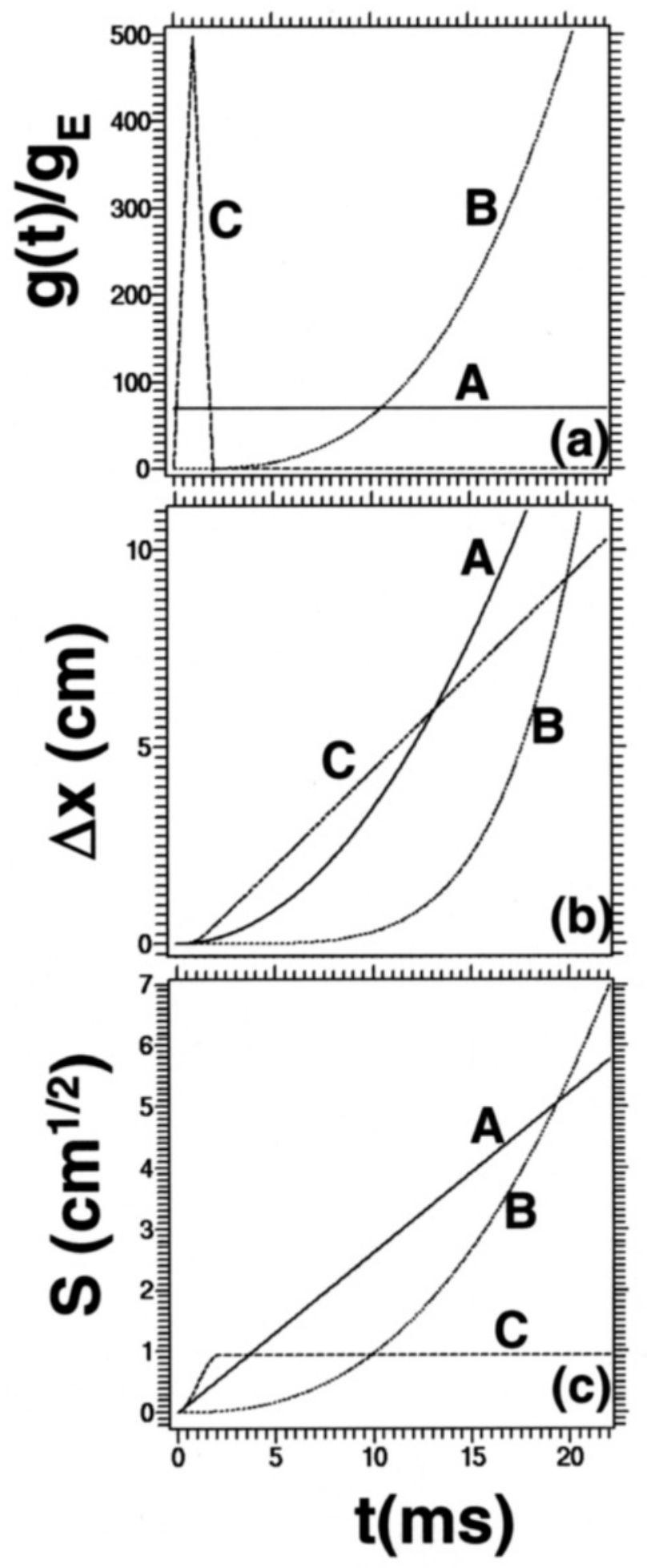

Fig. 13 


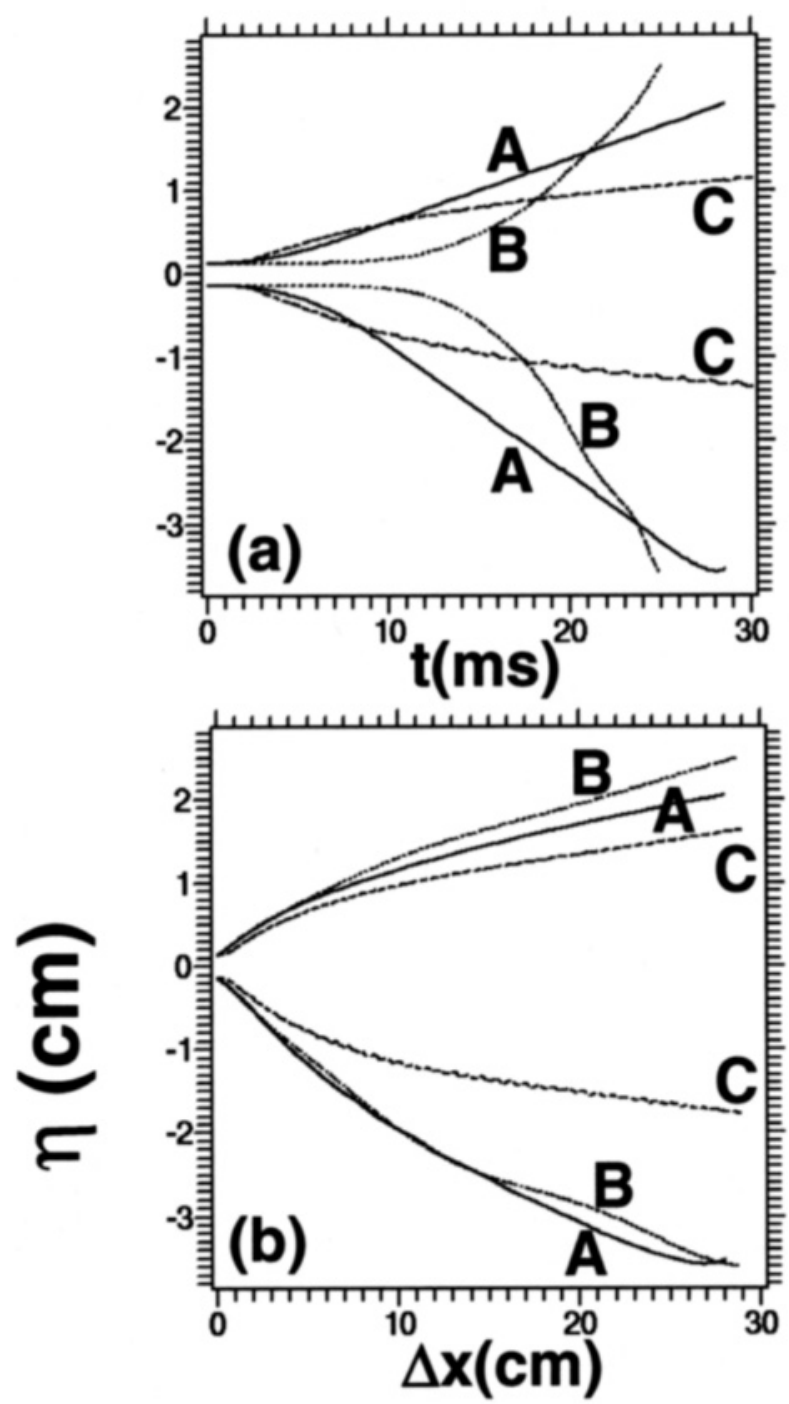

Fig. 14

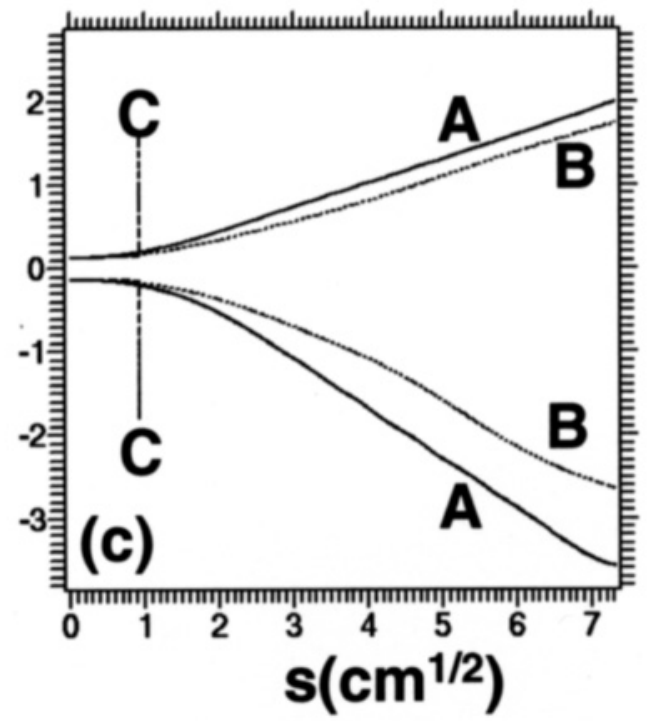



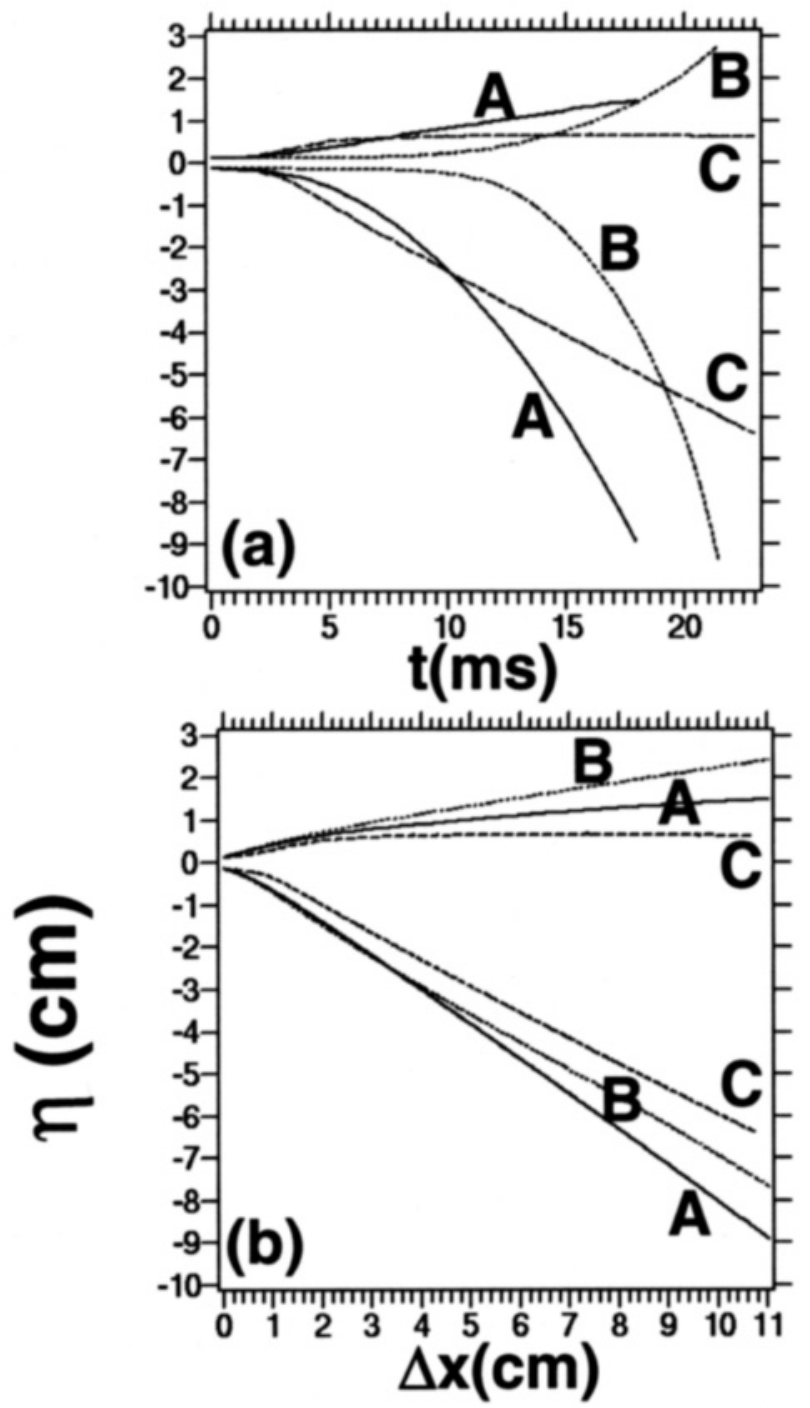

Fig. 15

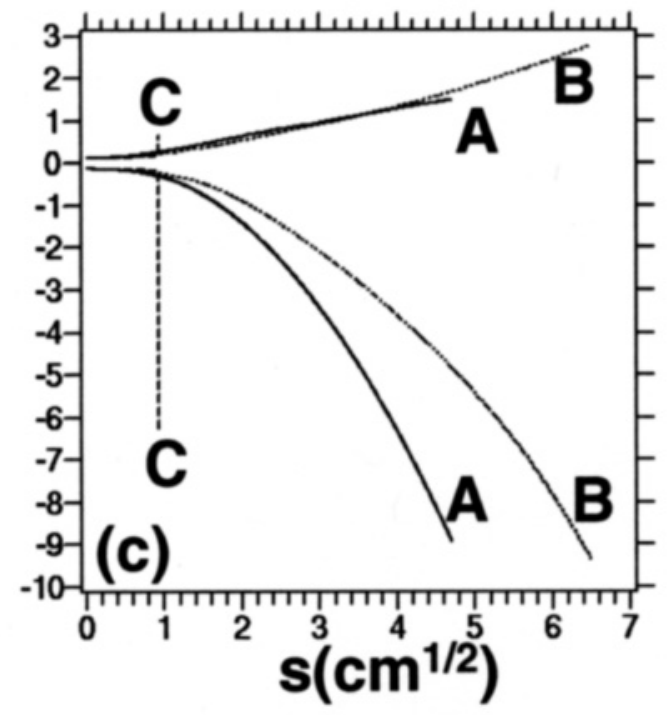




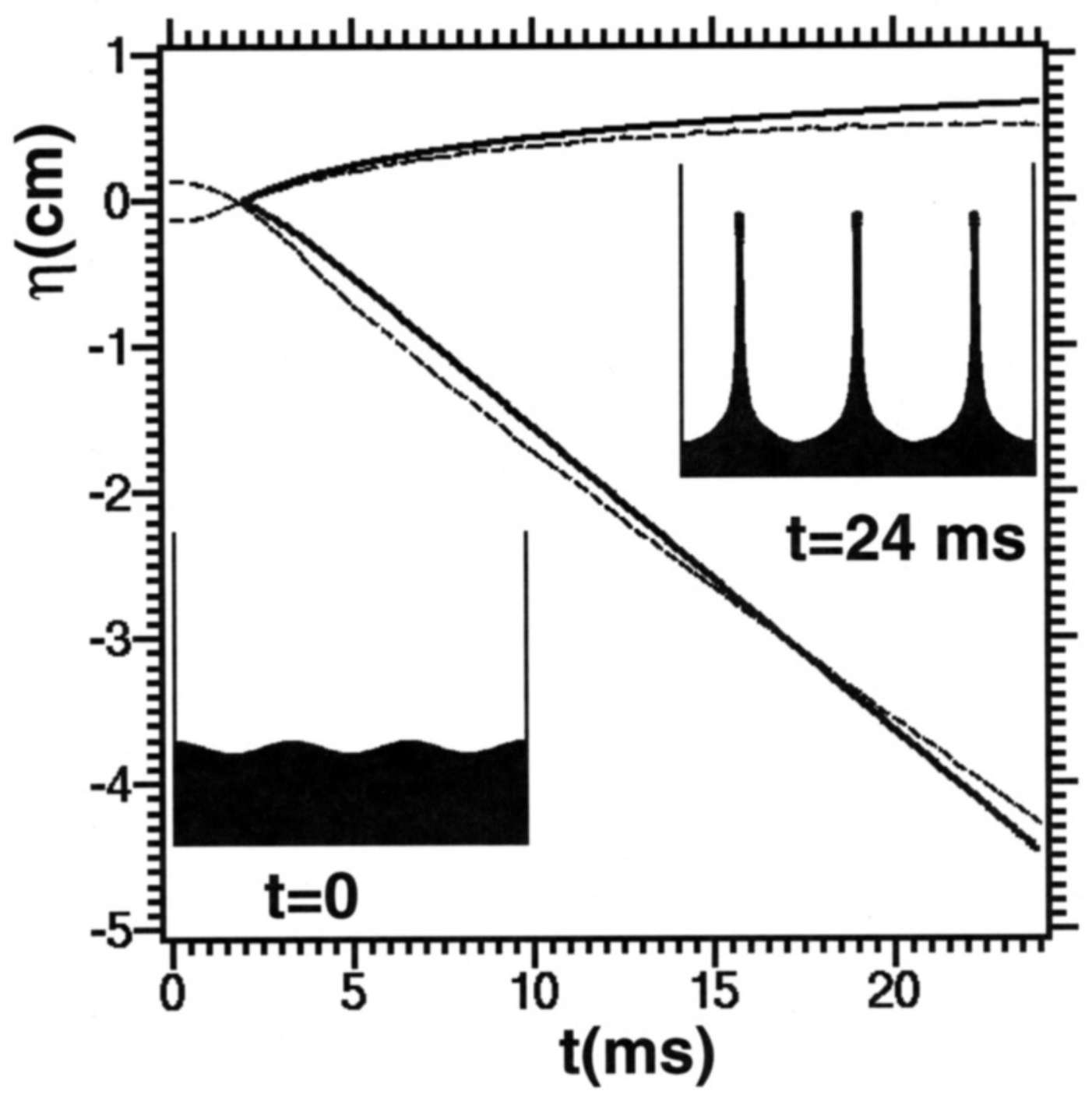

Fig. 16 


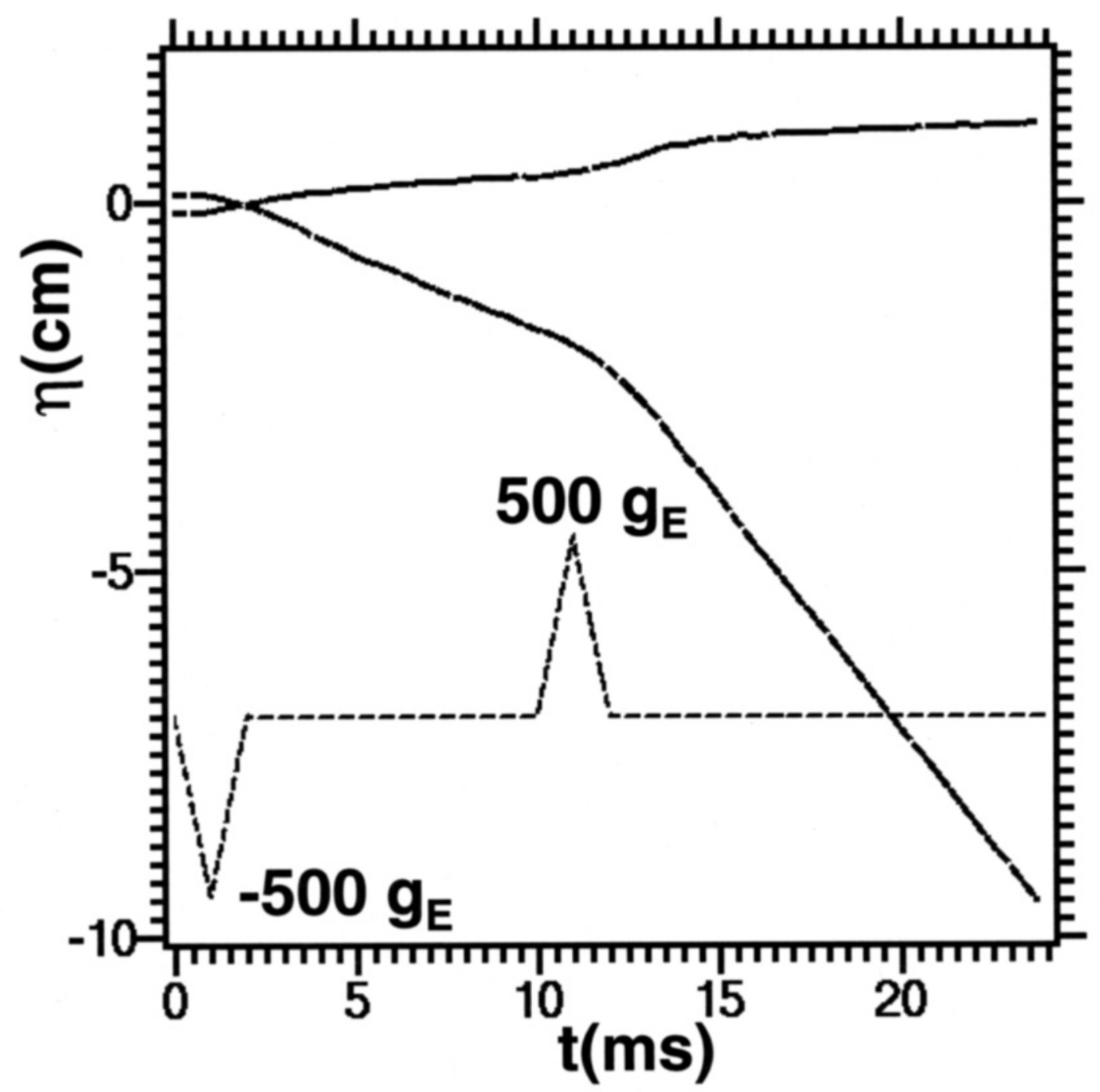

Fig. 17 


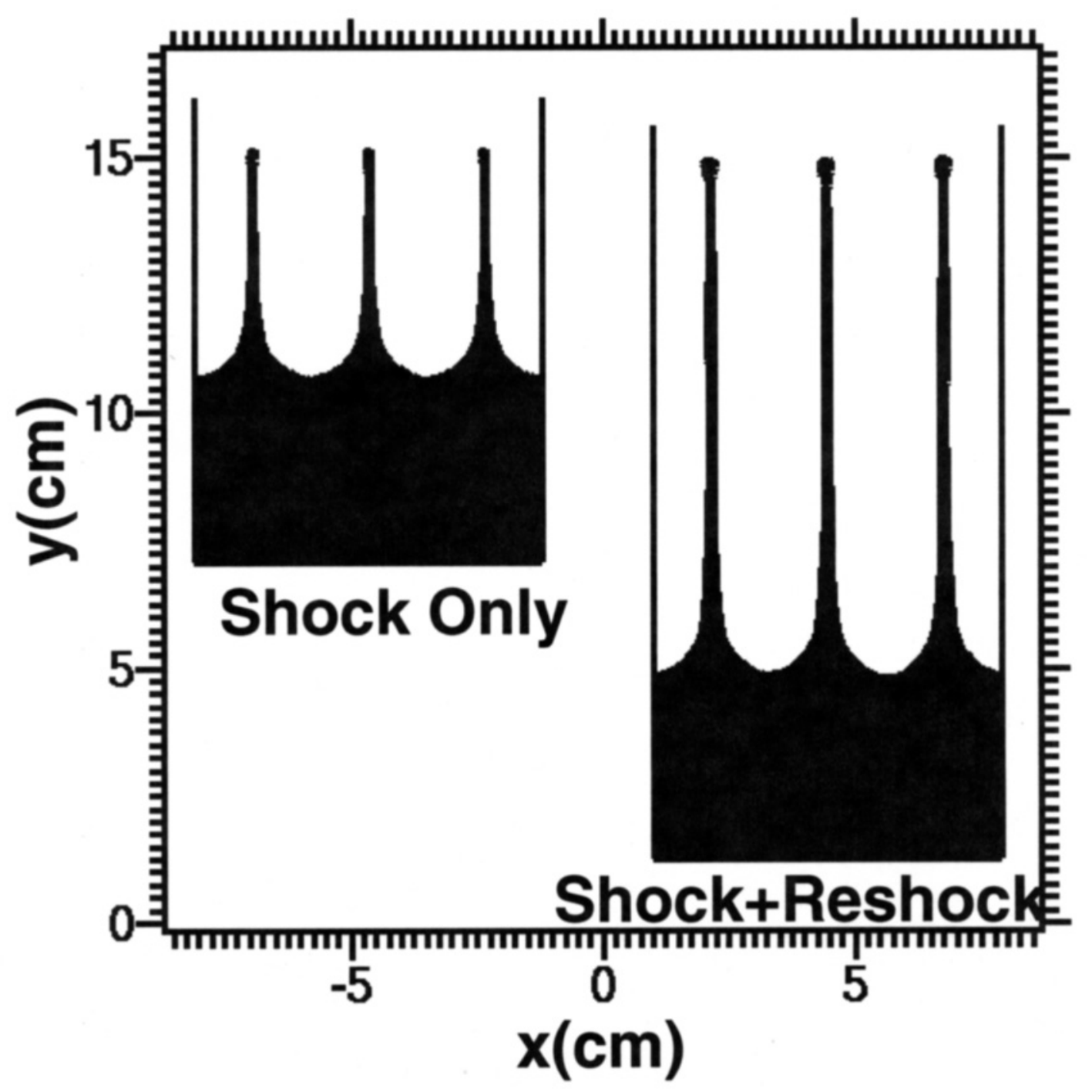

Fig. 18 